Published in "Geometriae Dedicata 183(1): 143-167, 2016"

which should be cited to refer to this work.

\title{
On commensurable hyperbolic Coxeter groups
}

\author{
Rafael Guglielmetti ${ }^{1}$ - Matthieu Jacquemet ${ }^{1}$. \\ Ruth Kellerhals ${ }^{1}$
}

\begin{abstract}
For Coxeter groups acting non-cocompactly but with finite covolume on real hyperbolic space $\mathbb{H}^{n}$, new methods are presented to distinguish them up to (wide) commensurability. We exploit these ideas and determine the commensurability classes of all hyperbolic Coxeter groups whose fundamental polyhedra are pyramids over a product of two simplices of positive dimensions.
\end{abstract}

Keywords Commensurability · Hyperbolic Coxeter group - Coxeter pyramid . Amalgamated free product $\cdot$ Translational lattice $\cdot$ Arithmetic group

Mathematics Subject Classification $51 \mathrm{~F} 15 \cdot 22 \mathrm{E} 40$

\section{Introduction}

Consider the real hyperbolic space $\mathbb{H}^{n}$ and its isometry group Isom $\left(\mathbb{H}^{n}\right)$. A hyperbolic Coxeter group of rank $N$ is a discrete subgroup of Isom $\left(\mathbb{H}^{n}\right)$ generated by $N$ reflections in hyperplanes of $\mathbb{H}^{n}$. These groups play an important role in different contexts of geometry and topology, in particular in lower dimensions. Although cofinite hyperbolic Coxeter groups can not exist anymore for dimensions $n \geq 996$, the classification of all these groups (or of large families of them) is an unresolved problem. In this work, we classify a large family of them up to wide commensurability. Here, two groups in $\operatorname{Isom}\left(\mathbb{H}^{n}\right)$ are commensurable in the wide

In memoriam Colin Maclachlan.

$\bowtie$ Ruth Kellerhals

ruth.kellerhals@unifr.ch

Rafael Guglielmetti

rafael.guglielmetti@unifr.ch

Matthieu Jacquemet

jacquemet.matthieu@gmail.com

1 Department of Mathematics, University of Fribourg, 1700 Fribourg, Switzerland 
sense (or commensurable, for brevity) if the intersection of one group with some conjugate of the other group is of finite index in both groups. This commensurability relation is an equivalence relation which preserves properties such as cocompactness, cofiniteness and arithmeticity.

In the planar case, Takeuchi [28] classified up to commensurability all arithmetic subgroups of $\operatorname{PSL}(2, \mathbb{R})$, that is, the group of orientation preserving isometries of $\mathbb{H}^{2}$. It turns out that there is only one commensurability class of non-cocompact but cofinite arithmetic hyperbolic Coxeter groups, and it is represented by the modular group $\operatorname{PSL}(2, \mathbb{Z})$. For dimensions $n \geq 3$, such a uniqueness result does not hold anymore, regardless of arithmeticity. In [14], the simplest family of hyperbolic Coxeter groups, that is, those of rank $N=n+1$, having a simplex fundamental domain, were classified up to commensurability. Observe that these groups exist for $n \leq 9$, only.

Consider discrete Coxeter groups in $\operatorname{Isom}\left(\mathbb{H}^{n}\right)$ generated by $N=n+2$ reflections in hyperplanes bounding a pyramid $P \subset \mathbb{H}^{n}$ of finite volume such that the neighborhood of the apex is a product of two simplices of positive dimensions. Each such pyramid is a non-compact Coxeter polyhedron whose apex is a point at infinity and belongs to a family of Coxeter polyhedra which was classified by Tumarkin in 2004 [29,30]. This set contains many polyhedra of dimensions $n$ up to 17 (see the "Appendix") and forms an important substitute for Coxeter simplices when $n>9$. Furthermore, this set provides both arithmetic and non-arithmetic reflection groups, and it furnishes the overall minimal covolume arithmetic discrete group related to the even modular $P S O\left(\mathrm{II}_{17,1}\right)$ in the orientation-preserving setting (see Fig. 2). We call them here hyperbolic Coxeter pyramid groups.

In this work, we classify hyperbolic Coxeter pyramid groups up to commensurability. To this end, we develop new methods of algebraic and geometrical nature. One important feature is the appearance of affine Coxeter subgroups coming along with Bieberbach's result about the existence of a finite index translational lattice of full rank. Another major aspect showing up for some pyramid groups is the free product structure with an amalgamated hyperbolic Coxeter simplex group. A result of KarrassSolitar about finite index subgroups in amalgamated free products will be of relevance. We also make use of other tools such as fields generated by traces of Coxeter elements.

By Vinberg's criterion, we can easily divide the family of Coxeter pyramid groups into its arithmetic and non-arithmetic constituents. For one pair of non-arithmetic hyperbolic Coxeter pyramid groups in $\operatorname{Isom}\left(\mathbb{H}^{3}\right)$, the discreteness of the commensurator of a nonarithmetic group and certain related volume comparisons (of high accuracy) allow us to conclude the desired result. The classification of non-arithmetic hyperbolic Coxeter pyramid groups is presented in Sect. 4.1. For arithmetic discrete Coxeter groups in $\operatorname{Isom}\left(\mathbb{H}^{n}\right)$ with $n=3$ resp. $n \geq 3$, one has the commensurability results of Maclachlan and Reid [23] resp. Maclachlan [21]. Our considerations are based on a complete invariant based on quaternion algebras. In Sect. 4.2, we give a brief but self-contained explanation of the commensurability classification and identify the pyramidal commensurability classes with the ones associated to arithmetic hyperbolic Coxeter simplices whenever possible. In this way, we round the classification of hyperbolic Coxeter pyramid groups up to commensurability.

Finally, let us add that our methods are suitable to study commensurability of more general hyperbolic groups. 


\section{Hyperbolic Coxeter groups and Coxeter polyhedra}

\subsection{Background}

Denote by $\mathbb{X}^{n}$ one of the three standard geometric $n$-spaces, the Euclidean space $\mathbb{E}^{n}$, the sphere $\mathbb{S}^{n}$, or the hyperbolic space $\mathbb{H}^{n}$, together with its isometry group $\operatorname{Isom}\left(\mathbb{X}^{n}\right)$. As in the spherical case, embed $\mathbb{H}^{n}$ in a quadratic space $\mathbb{Y}^{n+1}$ by interpreting $\mathbb{H}^{n}$ in the LorentzMinkowski space $\mathbb{E}^{n, 1}=\left(\mathbb{R}^{n+1},\langle x, y\rangle_{n, 1}=\sum_{i=1}^{n} x_{i} y_{i}-x_{n+1} y_{n+1}\right)$ of signature $(n, 1)$, that is,

$$
\mathbb{H}^{n}=\left\{x \in \mathbb{E}^{n, 1} \mid\langle x, x\rangle_{n, 1}=-1, x_{n+1}>0\right\}
$$

(see [32], for example). Then, the group $\operatorname{Isom}\left(\mathbb{H}^{n}\right)$ coincides with the group $P O(n, 1)$ of positive Lorentz-matrices. In the Euclidean case, we take the affine point of view and write $\mathbb{Y}^{n+1}=\mathbb{E}^{n} \times\{0\}$.

A subgroup $\Gamma \subset \operatorname{Isom}\left(\mathbb{X}^{n}\right)$ is a geometric Coxeter group if $\Gamma$ is a discrete group generated by finitely many reflections in hyperplanes of $\mathbb{X}^{n}$. The cardinality $N$ of the set of generators is called the rank of the group $\Gamma$.

Let $s_{i}$ be a generator of the geometric Coxeter group $\Gamma$ acting on $\mathbb{X}^{n}$ as the reflection with respect to the hyperplane $H_{i}(1 \leq i \leq N)$. Associate to $H_{i}$ a normal vector $e_{i} \in \mathbb{Y}^{n+1}$ of norm 1 such that

$$
H_{i}=\left\{x \in \mathbb{X}^{n} \mid\left\langle x, e_{i}\right\rangle_{\mathbb{Y}^{n+1}}=0\right\},
$$

and which bounds two closed half-spaces, for example,

$$
H_{i}^{-}=\left\{x \in \mathbb{X}^{n} \mid\left\langle x, e_{i}\right\rangle_{\mathbb{Y}^{n+1}} \leq 0\right\} .
$$

Since $\Gamma$ is a discrete subgroup of $\operatorname{Isom}\left(\mathbb{X}^{n}\right)$, it has a convex (closed) fundamental domain $P=P_{\Gamma} \subset \mathbb{X}^{n}$, and it can be supposed to be of the polyhedral form

$$
P=\bigcap_{i=1}^{N} H_{i}^{-} .
$$

The combinatorial, metrical and arithmetical properties of $P$ and simultaneously of $\Gamma$ can be read off from the Gram matrix $G(P)$ of $P$ formed by the products $\left\langle e_{i}, e_{j}\right\rangle_{\mathbb{Y}^{n+1}}(1 \leq i, j \leq$ $N)$. For more details and proofs, we refer to Vinberg's work [32]. Of particular interest is the hyperbolic case where the product $\left\langle e_{i}, e_{j}\right\rangle_{n, 1}$ characterises the mutual position of the hyperplanes $H_{i}, H_{j}$ as follows.

$$
-\left\langle e_{i}, e_{j}\right\rangle_{n, 1}= \begin{cases}\cos \frac{\pi}{m_{i j}} & \text { if } H_{i}, H_{j} \text { intersect at the angle } \frac{\pi}{m_{i j}} \text { in } \mathbb{H}^{n}, \\ 1 & \text { if } H_{i}, H_{j} \text { meet at } \partial \mathbb{H}^{n}, \\ \cosh l_{i j} & \text { if } H_{i}, H_{j} \text { are at distance } l_{i j} \text { in } \mathbb{H}^{n} .\end{cases}
$$

In particular, a fundamental domain $P \subset \mathbb{X}^{n}$ as in (2.4) for a geometric Coxeter group is a Coxeter polyhedron, that is, a polyhedron in $\mathbb{X}^{n}$ all of whose dihedral angles are submultiples of $\pi$. Conversely, each Coxeter polyhedron in $\mathbb{X}^{n}$ gives rise to a geometric Coxeter group.

The geometric Coxeter group $\Gamma$ has the presentation

$$
\Gamma=\left\langle s_{1}, \ldots, s_{N} \mid s_{i}^{2},\left(s_{i} s_{j}\right)^{m_{i j}}\right\rangle
$$

where $m_{i j}=m_{j i} \geq 2$ for $i \neq j$.

We restrict our attention to cocompact or cofinite geometric Coxeter groups, that is, we assume that the associated Coxeter polyhedra are compact or of finite volume in $\mathbb{X}^{n}$. 
In particular, hyperbolic Coxeter polyhedra are bounded by at least $n+1$ hyperplanes, appear as the convex hull of finitely many points in the extended hyperbolic space $\mathbb{H}^{n} \cup \partial \mathbb{H}^{n}$ and are acute-angled (no obtuse dihedral angles). An (ordinary) vertex $p \in \mathbb{H}^{n}$ of $P$ is given by a positive definite principal submatrix of rank $n$ of the Gram matrix $G(P)$ of $P$. Its vertex link $P_{p}$ is an $(n-1)$-dimensional spherical Coxeter polyhedron which is a product of $k \geq 1$ pairwise orthogonal lower dimensional spherical Coxeter simplices. A vertex (at infinity) $q \in \partial \mathbb{H}^{n}$ of $P$ is characterised by a positive semi-definite principal submatrix of rank $n-1$ of the Gram matrix $G(P)$. Its vertex link $P_{q}$ is a compact $(n-1)$-dimensional Euclidean Coxeter polyhedron which is a product of $l \geq 1$ pairwise orthogonal lower dimensional Euclidean Coxeter simplices. The polyhedron $P_{q}$ is a fundamental domain of the stabiliser $\Gamma_{q}<\operatorname{Isom}\left(\mathbb{E}^{n-1}\right)$ of $q$ which is a Bieberbach group containing a finite index translational lattice of rank $n-1$. In order to measure the translational length of a generating translation in $\Lambda$, we pass to the upper half space model $\left(\mathbb{E}_{+}^{n}, d s^{2}=\left(d x_{1}^{2}+\cdots+d x_{n}^{2}\right) / x_{n}^{2}\right)$ for $\mathbb{H}^{n}$ and assume that $q=\infty$. In this way, the polyhedron $P_{\infty}$ can be seen as lying on the (canonical) horosphere at height $x_{n}=1$ so that edge lengths are measured by means of the (undistorted) Euclidean line element

$$
d s_{0}^{2}=d x_{1}^{2}+\cdots+d x_{n-1}^{2} .
$$

For the arithmeticity test of a hyperbolic Coxeter group $\Gamma$ with associated Gram matrix $G(P)$, there is a powerful criterion due to Vinberg (see [34, pp. 226-227]). In the special case of a cofinite but non-cocompact group $\Gamma$, it can be stated as follows. Write $2 G(P)=:\left(g_{i j}\right)$, and consider cycles (of length $k$ ) of the form

$$
g_{i_{1} i_{2}} g_{i_{2} i_{3}} \cdots g_{i_{k-1} i_{k}} g_{i_{k} i_{1}},
$$

with distinct indices $i_{j}$ in $2 G(P)$. Then, $\Gamma$ is arithmetic with field of definition $\mathbb{Q}$ if and only if all the cycles of $2 G(P)$ are rational integers.

Many of these features can be visualised by the Coxeter graph $\Sigma$ of $P$ and $\Gamma$. To each hyperplane $H_{i}$ of $P$ and to each generator $s_{i} \in \Gamma$ corresponds a node $v_{i}$ of $\Sigma$. Two nodes $v_{i}, v_{j}$ are joined by an edge with label $m_{i j} \geq 3$ if $\measuredangle\left(H_{i}, H_{j}\right)=\pi / m_{i j}$ (the label 3 is usually omitted). If $H_{i}, H_{j}$ are orthogonal, their nodes are not connected. If $H_{i}, H_{j}$ meet at $\partial \mathbb{H}^{n}$, their nodes are joined by a bold edge (or by an edge with label $\infty$ ); if they are at distance $l_{i j}>0$ in $\mathbb{H}^{n}$, their nodes are joined by a dotted edge, mostly without the label $\cosh l_{i j}$. We will also use the Coxeter symbol for a Coxeter group. For example, $[p, q, r]$ is associated to a linear Coxeter graph with 3 edges of consecutive labels $p, q, r$, and the Coxeter symbol $[(p, q, r)]$ describes a cyclic graph with labels $p, q, r$. Sometimes, we abbreviate even further and write $\left[p^{2}\right]$ instead of $[p, p]$, and so on. The Coxeter symbol $\left[3^{i, j, k}\right]$ denotes a group with Y-shaped Coxeter graph with strings of $i, j$ and $k$ edges emanating from a common node. We assemble the different symbols into a single one in order to describe the different nature of parts of the Coxeter graph in question (see Fig. 1 and also [13]).

Suppose that $\Gamma$ is a cofinite but non-cocompact hyperbolic Coxeter group such that its graph $\Sigma$ contains no dotted edges. Then, by a result of Guglielmetti [8, Proposition 1.13], $\Gamma$ is arithmetic if and only if all labels of $\Sigma$ lie in $\{\infty, 2,3,4,6\}$ and each cycle of length at least 3 in $2 G(P)$ lies in $\mathbb{Z}$.

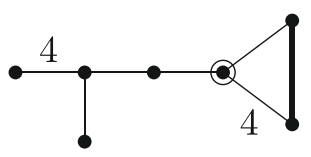

Fig. 1 The Coxeter pyramid group $\left[4,3^{1,2},(3, \infty, 4)\right]$ acting on $\mathbb{H}^{5}$ 
The program CoxIter [8] developped by Guglielmetti allows us to exploit Vinberg's results [32] and to determine whether a Coxeter group $\Gamma \subset \operatorname{Isom}\left(\mathbb{H}^{n}\right)$ is cocompact or cofinite and whether it is arithmetic. Furthermore, it provides the combinatorial structure in form of the $f$-vector of $P_{\Gamma}$, the Euler characteristic and the covolume if, in the latter case, $n$ is even. More concretely, for $n$ even, the covolume of $\Gamma \subset \operatorname{Isom}\left(\mathbb{H}^{n}\right)$ is given by (see [9])

$$
\operatorname{covol}_{n} \Gamma=\operatorname{vol}_{n}\left(P_{\Gamma}\right)=\frac{(2 \pi)^{\frac{n}{2}}}{1 \cdot 3 \cdot \ldots \cdot(2 n-1)} \cdot|\chi(\Gamma)| .
$$

While the irreducible spherical and Euclidean Coxeter groups are well known (for the corresponding lists, see [34, pp. 202-203], for example), hyperbolic Coxeter groups are far from being classified. It is known that Coxeter groups acting cofinitely (resp. cocompactly) on $\mathbb{H}^{n}$ do not exist for $n \geq 996$ (resp. $n \geq 30$ ). There are partial classification results for groups of small rank $N$ and for certain families of arithmetic groups. In particular, the hyperbolic Coxeter simplex groups, characterised by $N=n+1$, were classified by Lannér in the cocompact case and by Koszul in the non-cocompact case; they exist up to $n=9$. In $[13,14]$, the covolumes and commensurability classes of all cofinite hyperbolic simplex groups were determined.

\subsection{Hyperbolic Coxeter groups with $\mathbf{n}+2$ generators}

Consider a Coxeter group with $N=n+2$ generators acting cofinitely on $\mathbb{H}^{n}$ such that the associated Coxeter polyhedron is combinatorially a pyramid over a product of two simplices of positive dimensions. Such a pyramid is not compact since its apex, with a neighborhood being a cone over a product of two Euclidean simplices, has to be a point at infinity. In the associated Coxeter graph $\Sigma$, the node separating $\Sigma$ into the two disjoint corresponding Euclidean Coxeter subgraphs is encircled (for more details, see [29, Section 4], [30, Section 4]). In 2004, Tumarkin [29,30] classified this family of hyperbolic pyramid groups by using Gale diagram techniques. The list comprises exactly 200 groups and is given in condensed form in the "Appendix". From his list follows that the groups exist up to dimension $n=17$, comprising non-arithmetic examples up to dimension 10 (see also [33]), as well as a single but very distinguished group $\Gamma_{*}$ in dimension 17 .

In fact, the group $\Gamma_{*}$ is closely related to the even unimodular group $P S O\left(\mathrm{II}_{17,1}\right)$ which, by a result of Emery [4, Theorem 1], is the fundamental group of the (unique up to isometry) hyperbolic $n$-space form of minimal volume among all orientable arithmetic hyperbolic $n$ orbifolds for $n \geq 2$. The Coxeter graph of $\Gamma_{*}$ is given in Fig. 2, and the associated Coxeter polyhedron $P_{*}$ is of volume (see [4, Section 3])

$$
\operatorname{vol}_{17}\left(P_{*}\right)=\operatorname{vol}_{17}\left(\mathbb{H}^{17} / P S O\left(\mathrm{II}_{17,1}\right)\right)=\frac{691 \cdot 3617}{2^{38} \cdot 3^{10} \cdot 5^{4} \cdot 7^{2} \cdot 11 \cdot 13 \cdot 17} \zeta(9) .
$$

The top dimensional non-arithmetical Coxeter pyramid group $\Gamma_{3,4} \subset \operatorname{Isom}\left(\mathbb{H}^{10}\right)$ is given by the Coxeter symbol $\left[3^{2,1}, 3^{6},(3, \infty, 4)\right]$ and by the Coxeter graph in Fig. 3 (for $k=3, l=$ 4). It turns out to be the free product with amalgamation $\Gamma_{3,4}=\widehat{\Gamma}_{3} \star_{\Phi} \widehat{\Gamma}_{4}$ of the two (noncommensurable) arithmetic Coxeter groups $\widehat{\Gamma}_{l}=\left[3^{2,1}, 3^{6}, l\right], l=3,4$, of infinite covolume

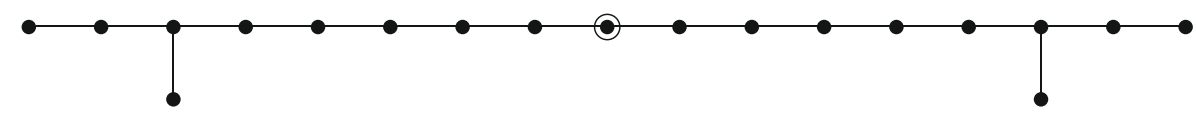

Fig. 2 The graph of the Coxeter pyramid $P_{*}=\left[3^{2,1}, 3^{12}, 3^{1,2}\right]$ in $\mathbb{H}^{17}$ 


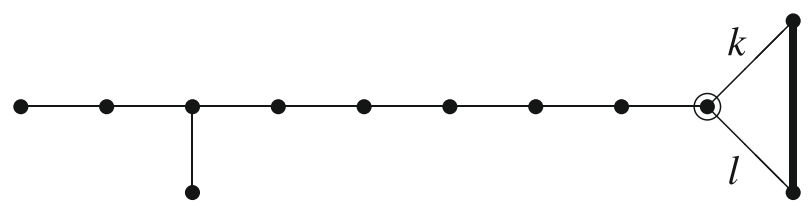

Fig. 3 The Coxeter pyramid groups $\Gamma_{k, l} \subset \operatorname{Isom}\left(\mathbb{H}^{10}\right)$

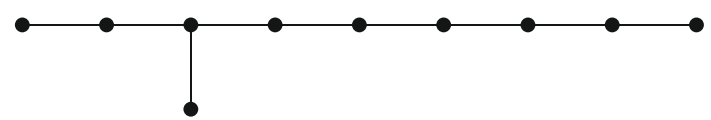

Fig. 4 The common subgroup $\Phi$ of $\Gamma_{2,3}$ and $\Gamma_{2,4}$

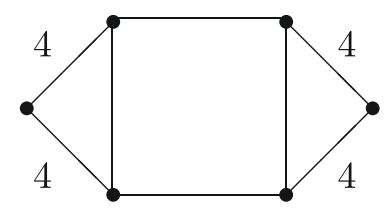

Fig. 5 The Coxeter group $\widetilde{\Gamma} \subset \operatorname{Isom}\left(\mathbb{H}^{4}\right)$

(see Fig. 3; cf. [33]); the group $\Gamma_{3,4}$ is a mixture in the sense of Gromov and Piatetski-Shapiro [7] and has a Coxeter polyhedron $P_{3,4} \subset \mathbb{H}^{10}$ which is obtained by glueing the Coxeter pyramids $P_{2,3}, P_{2,4}$ with Coxeter graphs $\Gamma_{2,3}=\left[3^{2,1}, 3^{6}, 3, \infty\right], \Gamma_{2,4}=\left[3^{2,1}, 3^{6}, 4, \infty\right]$ along their common Coxeter facet $F \subset \mathbb{H}^{9}$ with reflection group $\Phi$ and Coxeter graph given by Fig. 4.

The set of all (up to finite index) non-arithmetic hyperbolic Coxeter pyramid groups with $n+2$ generators is treated in Sect. 4.1. Together with Tumarkin's arithmetic Coxeter group $\widetilde{\Gamma} \subset \operatorname{Isom}\left(\mathbb{H}^{4}\right)$, depicted in Fig. 5, and whose Coxeter polyhedron is a product of two triangles, the Coxeter pyramid groups form the class of all cofinite and non-cocompact hyperbolic Coxeter groups with $n+2$ generators in $\operatorname{Isom}\left(\mathbb{H}^{n}\right)$. Notice that the associated Gram matrices are of signature $(n, 1,1)$ and not invertible.

In all cases, the Coxeter pyramid groups are - up to finite index - reflection groups whose fundamental domains are polarly (or totally orthogonally) truncated simplices. As an example, the pyramid $P_{*} \subset \mathbb{H}^{17}$ (see Fig. 2) is a polarly truncated simplex whose description together with inradius and local density are given in [11, p. 1015]. This property-in a more restricted context (see Lemma 1)—will be very useful when studying the commensurability of certain non-arithmetic Coxeter pyramid groups which are free products of two groups $\widehat{\Gamma}_{k}=\left[p_{1}, p_{2}, \ldots, p_{n-1}, q_{k}\right], k=1,2$, amalgamated by the Coxeter simplex group $\Phi$ with symbol $\left[p_{1}, p_{2}, \ldots, p_{n-1}\right]$, with $p_{1}=\infty$ for $n=3$. Here, each of the involved Coxeter groups $\widehat{\Gamma}_{k}$ gives rise to a hyperbolic Coxeter pyramid which is combinatorially a simply truncated simplex with the truncating polar hyperplane $H_{u}$, associated to one ultra-ideal vertex $u \notin \mathbb{H}^{n} \cup \partial \mathbb{H}^{n}$, intersecting the hyperplane opposite to $u$ at the point at infinity which is the apex of the pyramid. Algebraically, we get the following interpretation of the Coxeter pyramid group $\Gamma:=\left[p_{1}, p_{2}, \ldots, p_{n-1},\left(q_{1}, \infty, q_{2}\right)\right]$. Denote by $s=s(k, u)$ the reflection with respect to the hyperplane $H_{u}$, and let $\langle s\rangle$ be the normal closure of $s$ in the group $\widehat{\Gamma}_{k}$. Then, we have $\Gamma_{k}=\widehat{\Gamma}_{k} \ltimes\langle s\rangle$, and $\Gamma=\left[p_{1}, p_{2}, \ldots, p_{n-1},\left(q_{1}, \infty, q_{2}\right)\right]=\widehat{\Gamma}_{1} \star_{\Phi} \widehat{\Gamma}_{2}$. A concrete example is given by the Coxeter pyramid group represented by the graph $[\infty, 3,(3, \infty, 5)]$, which is the free product of the Coxeter tetrahedral groups $[\infty, 3,3]$ and $[\infty, 3,5]$ amalgamated by their common subgroup $[\infty, 3]$ (see Fig. 6). 


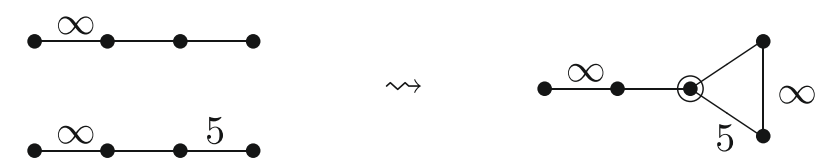

Fig. 6 The pyramid group $[\infty, 3,(3, \infty, 5)]$ as free product amalgamated by $[\infty, 3]$
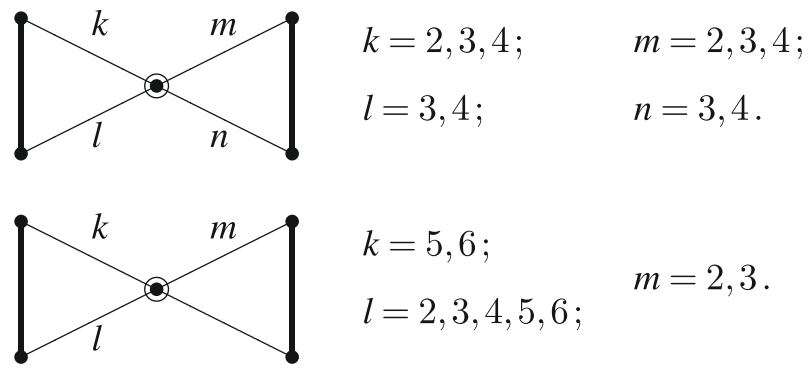

Fig. 7 The Coxeter pyramids $[(k, \infty, l),(m, \infty, n)]$ and $[(k, \infty, l),(m, \infty, 3)]$ in $\mathbb{H}^{3}$
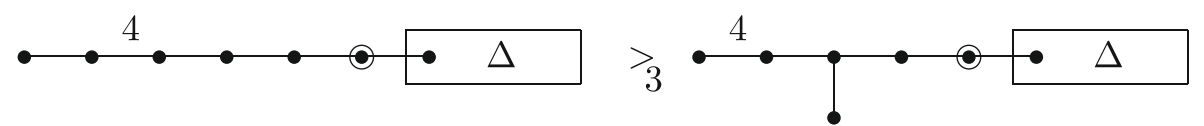

Fig. 8 The group $\Gamma=\left[\widetilde{F}_{4}, 3,3, \Delta\right]$ and its subgroup $\left[\widetilde{B}_{4}, 3,3, \Delta\right]$ of index 3

For the Coxeter pyramid groups $\Gamma_{m, n}^{k, l} \subset \operatorname{Isom}\left(\mathbb{H}^{3}\right)$ given by the Coxeter graphs according to Fig. 7, there is a closed formula (up to minor sign errors), due to Vinberg [34, pp. 129-130], for the covolume in terms of Lobachevsky's function

$$
Л(\omega)=\frac{1}{2} \sum_{r=1}^{\infty} \frac{\sin (2 r \omega)}{r^{2}}=-\int_{0}^{\omega} \log |2 \sin t| d t, \quad \omega \in \mathbb{R} .
$$

In particular, the non-arithmetic Coxeter pyramid groups $\Gamma_{3,4}^{2,3}$ and $\Gamma_{3,4}^{2,4}$ are of covolumes

$$
\begin{aligned}
& \operatorname{covol}_{3}\left(\Gamma_{3,4}^{2,3}\right)=\frac{1}{3} Л\left(\frac{\pi}{4}\right)+\frac{1}{8} \cdot\left(\frac{\pi}{6}\right)+Л\left(\frac{5 \pi}{24}\right)-Л\left(\frac{\pi}{24}\right) \simeq 0.40362118, \\
& \operatorname{covol}_{3}\left(\Gamma_{3,4}^{2,4}\right)=Л\left(\frac{\pi}{4}\right)+\frac{1}{8} \cdot\left(\frac{\pi}{6}\right)+Л\left(\frac{5 \pi}{24}\right)-Л\left(\frac{\pi}{24}\right) \simeq 0.70894305 .
\end{aligned}
$$

As for finite index subgroup relations between Coxeter groups, there are only few general criteria, for example those due to Maxwell [24, Proposition 3.1, Proposition 3.2]. Applied to a Coxeter pyramid group $\Gamma$ with Euclidean Coxeter subgroups of type $\widetilde{F}_{4}=[3,4,3,3]$ and $\Delta$ (see Fig. 8 for the Coxeter graph $\Sigma$ of $\Gamma$ ), Maxwell's result shows that the hyperbolic Coxeter pyramid group with Euclidean Coxeter subgroups $\widetilde{B}_{4}=\left[4,3,3^{1,1}\right]$ (replacing $\widetilde{F}_{4}$ ) and $\Delta$ is a subgroup of index 3 in $\Gamma$.

There are also some ad hoc results based on looking at additional hyperplanes bisecting Coxeter pyramids into Coxeter polyhedra or at Coxeter groups related to higher Bianchi groups. In this way, in dimension 3 , a natural bisection shows that the Coxeter pyramid groups $[\infty, 3,3, \infty]$ resp. $[\infty, 4,4, \infty]$ are subgroups of index 2 in the Coxeter simplex groups [3, 4, 4] resp. [4, 4, 4], and the latter group is related to the last but one by an index 3 subgroup relation arising by a tetrahedral trisection. As an example in dimension 4 , by $[15$, 


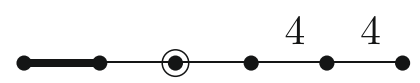

Fig. 9 The Coxeter pyramid group $[\infty, 3,3,4,4]$ acting on $\mathbb{H}^{4}$

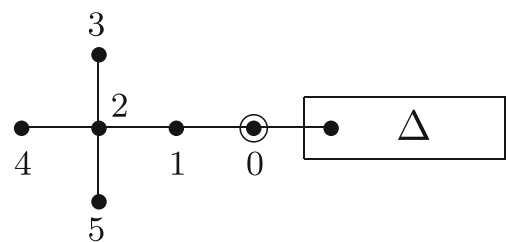

Fig. 10 The group $\Gamma$ with Euclidean Coxeter subgroups $\widetilde{D}_{4}$ and $\Delta$

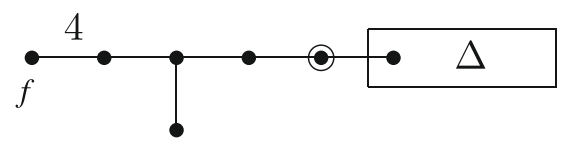

Fig. 11 The hyperbolic Coxeter group $\Gamma_{f}$

p. 172], the Coxeter pyramid group [ $\infty, 3,3,4,4]$ given by Fig. 9 is a subgroup of index 3 in the Coxeter simplex group [3, 4, 3, 4]; the latter group generates the symmetry group of the right-angled ideal regular 24-cell and is also related to the quaternionic modular group $\operatorname{PSL}(2, \mathbb{H})$.

Let us add an example of how to determine a subgroup relation given implicitly by the symmetry of a Coxeter graph. Consider a hyperbolic Coxeter group $\Gamma$ with one Euclidean Coxeter subgroup $\Delta$ and with one Euclidean Coxeter subgroup, disconnected from $\Delta$, having a symmetric Coxeter graph such as the Coxeter triangle graph $[(k, l, k)]$ with integers $k \geq 3$ and $2 \leq l \leq \infty$, or $\widetilde{A}_{3}=\left[3^{[4]}\right]$ or $\widetilde{D}_{4}=\left[3,3^{1,1,1}\right]$. In the latter case, a hyperbolic realisation of such a graph $\Sigma$ of $\Gamma$ is given by Fig. 10. Interpret the nodes $i$ for $i=0, \ldots, 5$ as unit vectors $e_{i}$ normal to the mirror hyperplanes $H_{i}$ of the reflections $s_{i}$ in $\Gamma$ directing outwards with respect to the Coxeter polyhedron $P=P(\Gamma)$, say.

Consider the new unit vector $f=\left(e_{5}-e_{4}\right) / \sqrt{2}$ which is Lorentz-orthogonal to the bisecting hyperplane $H_{f}$ of $H_{4}$ and $H_{5}$. Denote by $t$ the reflection with respect to $H_{f}$ and compute the Lorentz products $\left\langle f, e_{i}\right\rangle$ for all $i \neq 5$ by taking into account the labels as given in Fig. 10. It follows that the Coxeter polyhedron $P$ associated to $\Gamma$ decomposes into two isometric copies of the Coxeter polyhedron with graph given by Fig. 11 and that $\Gamma$ is of index 2 in the associated Coxeter group $\Gamma_{f}$ (given by replacing $s_{5}$ by s).

\section{Commensurable hyperbolic Coxeter groups}

Two discrete subgroups $G_{1}, G_{2} \subset \operatorname{Isom}\left(\mathbb{H}^{n}\right)$ are said to be commensurable (in the wide sense-a specification which we shall omit in the sequel) if the intersection $G_{1} \cap G_{2}^{\prime}$ of $G_{1}$ with some conjugate $G_{2}^{\prime}$ of $G_{2}$ in $\operatorname{Isom}\left(\mathbb{H}^{n}\right)$ is of finite index both in $G_{1}$ and in $G_{2}^{\prime}$. In this case, and up to isometry, the associated orbifolds $\mathbb{H}^{n} / G_{1}$ and $\mathbb{H}^{n} / G_{2}$ admit a common finite sheeted cover.

Notice that the intersection $H:=G_{1} \cap G_{2}^{\prime}$ contains a non-trivial normal subgroup $N$ of finite index in $G_{1}$, the normal core of $H$, which is given by $\cap_{\gamma \in G_{1}} \gamma H \gamma^{-1}$. 
The commensurability relation is an equivalence relation which is stable when passing to a finite index subgroup. In particular, one can study commensurability on the level of orientation preserving subgroups (of index two) or-by Selberg's Lemma-by passing to a finite index torsion-free subgroup. This is advisable for dimension $n=2$ resp. $n=3$ where the group $P S O(n, 1)$ of orientation preserving isometries is isomorphic to $P S L(2, \mathbb{R})$ resp. $\operatorname{PSL}(2, \mathbb{C})$

It follows from the definition that the commensurability relation on the set $\mathcal{G}$ of discrete groups $G \subset \operatorname{Isom}\left(\mathbb{H}^{n}\right)$ preserves properties such as cocompactness, cofiniteness and arithmeticity.

An important characterisation of arithmeticity is due to Margulis (see [23, Theorem 10.3.5], for example). Consider the commensurator

$\operatorname{Comm}(G)=\left\{\gamma \in \operatorname{Isom}\left(\mathbb{H}^{n}\right) \mid G\right.$ and $\gamma G \gamma^{-1}$ is of finite index in $G$ and $\left.\gamma G \gamma^{-1}\right\}$

of a cofinite discrete group $G \subset \operatorname{Isom}\left(\mathbb{H}^{n}\right), n \geq 3$. Then, $\operatorname{Comm}(G) \subset \operatorname{Isom}\left(\mathbb{H}^{n}\right)$ is a discrete subgroup in $\operatorname{Isom}\left(\mathbb{H}^{n}\right)$ containing $G$ with finite index if and only if $G$ is nonarithmetic. In particular, for $G$ non-arithmetic, the commensurator $\operatorname{Comm}(G)$ is the maximal element in the commensurability class of $G$ so that all non-arithmetic hyperbolic orbifolds with fundamental groups commensurable to $G$ cover a smallest common quotient (up to isometry).

Suppose that a finite volume hyperbolic orbifold $Q=\mathbb{H}^{n} / G$ has a single cusp. In other words, the group $G$ has a fundamental polyhedron with precisely one ideal vertex $q \in \partial \mathbb{H}^{n}$. Then, the stabiliser $G_{q}<G$ is a crystallographic group containing a translational lattice $\Lambda \cong \mathbb{E}^{n-1}$ of finite index. Let $U_{q} \subset Q$ be the maximal embedded cusp neighborhood. Then, the quotient $\operatorname{vol}_{n}\left(U_{q}\right) / \operatorname{vol}_{n}(Q)$ is called the cusp density of $Q$. It is known that for 1-cusped orbifolds $Q=\mathbb{H}^{n} / G$ with discrete commensurator $\operatorname{Comm}(G)$, the cusp density is a commensurability invariant (see [6, Section 2]). However, since (transcendental) volume expressions are involved, this property is of limited value [see (2.9) and (2.10), for example]. In the case of 1-cusped quotients by non-arithmetic Coxeter pyramid groups (see Sect. 4.1) a more elementary reasoning without volume computation but based on crystallography (see Lemma 1 and Proposition 1) will be exploited.

In fact, volume considerations rarely help to judge about commensurability in a rigorous way but relate such questions to analytical number theory. For example, if the covolume quotient of two groups in the set in $\mathcal{G}$ is an irrational number, then the groups cannot be commensurable. Notice that this observation is void for $n=2 k$ since the covolume-via the Euler characteristic and the theorem of Gauss-Bonnet-is a rational multiple of $\pi^{k}$ (see 2.8).

For $n=2 k+1 \geq 3$, the covolume (in-)commensurability is connected to difficult number theoretical questions. In particular, for $n=3$, these questions are related to conjectures of Chowla, Milnor and others concerning the commensurability of generalised Hurwitz zeta values $\zeta(2, k / N)$ and-more specifically—about the $\mathbb{Q}$-linear independence of Lobachevsky values at arguments $k \pi / N$ with $k$ relatively prime to $N$ and $0<k<N / 2$ (see [2,26]).

A natural conjugacy invariant is the (ordinary) trace field $\operatorname{Tr}(G)$ of $G$ in $G L(n+1, \mathbb{R})$ which is defined to be the field generated by all the traces of matrices in $G \subset P O(n, 1)$. In particular, consider a hyperbolic Coxeter group $\Gamma$ of rank $N$ with generators $s_{1}, \ldots, s_{N}$ and a Coxeter element $c=s_{1} \ldots s_{N}$. Now, it is easy to see that each finite index subgroup of $\Gamma$ must contain $c^{k}$ for some positive integer $k$. Following ideas and methods in [14, p. 132], where all hyperbolic Coxeter simplex groups (of rank $N=n+1$ ) are classified up to commensurability, incommensurability for hyperbolic Coxeter groups of higher rank $N$ can be tested as follows: Consider two cofinite hyperbolic Coxeter groups $\Gamma_{1}, \Gamma_{2} \subset P O(n, 1)$ of equal rank $N$ with Coxeter elements $c_{1}$ and $c_{2}$, and let $T_{i}^{k}=\mathbb{Q}\left(\operatorname{tr}\left(c_{i}^{k}\right)\right) \subset \operatorname{Tr}\left(W_{i}\right), i=1,2$, 
$k \in \mathbb{N}^{*}$, be the fields generated by the traces of the $k$-th powers of $c_{1}$ and $c_{2}, k \in \mathbb{N}^{*}$. If

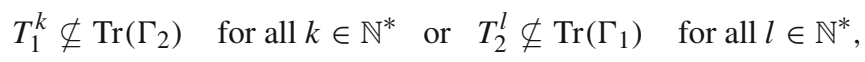

then $\Gamma_{1}$ and $\Gamma_{2}$ are not commensurable (for more details and examples in the case of hyperbolic Coxeter pyramids, see [12, pp. 71-73]).

In the particular case of Kleinian groups, that is, of cofinite discrete groups $G \subset$ $\operatorname{PSL}(2, \mathbb{C})$, one can sometimes avoid trace computations by considering the invariant trace field $k G=\mathbb{Q}\left(\operatorname{Tr}\left(G^{(2)}\right)\right)$, generated by the traces of all squared elements $\gamma^{2}, \gamma \in G$, and the invariant quaternion algebra $A G$ over $k G$. Both, the field $k G$ and the algebra $A G$ are commensurability invariants (see [27]). Furthermore, $k G$ is a finite non-real extension of $\mathbb{Q}$ (see [23, Theorem3.3.7]), and if the group $G$ is not cocompact (containing parabolic elements), then the algebra $A G$ is isomorphic to the matrix algebra $M_{2}(k G)$ (see [23, Theorem 3.3.8]).

In the case when the group $G$ is an amalgamated free product of the form $G=G_{1} \star_{H} G_{2}$, where $H$ is a non-elementary Kleinian group, then $k G$ is a composite of fields according to (see [23, Theorem 5.6.1])

$$
k G=k G_{1} \cdot k G_{2} .
$$

Now, in the special case of the rotational subgroup $\Gamma^{+} \subset P S L(2, \mathbb{C})$ of a cofinite hyperbolic Coxeter group $\Gamma \subset P O(3,1)$, Maclachlan and Reid [22, Theorem 3.1] provide a nice characterisation in terms of the Gram matrix $G(P)$ and a certain field $K(P)$. The field $K(P)$ is generated by the cycles in $2 G(P)=:\left(g_{i j}\right)$ of the associated Coxeter polyhedron $P \subset \mathbb{H}^{3}$, with normal vectors $e_{1}, \ldots, e_{N}$ directed outwards, of $\Gamma$ (see Sect. 2.1). In particular, the invariant trace field is given by

$$
k \Gamma^{+}=K(P)(\sqrt{d}),
$$

where $d$ is the discriminant of the linear space $V(P)$ of dimension 4 over $K(P)$, generated by the vector $v_{1}=2 e_{1}$ and

$$
v_{i_{1} i_{2} \ldots i_{k}}=g_{1 i_{1}} g_{i_{1} i_{2}} \cdots g_{i_{k-1} i_{k}} e_{i_{k}}, \quad\left\{i_{1}, i_{2}, \ldots, i_{k}\right\} \subset\{1, \ldots, N\},
$$

and equipped with the restriction $q_{V}$ of the form $\langle\cdot, \cdot\rangle_{3,1}$ to $V(P)$. In addition, for the invariant quaternion algebra $A \Gamma^{+}$, there is a similarly neat characterisation (for details, see [22, Theorem 3.1(iii)]).

In our work, commensurability criteria are needed for the family of Coxeter pyramid groups $\Gamma \subset \operatorname{Isom}\left(\mathbb{H}^{n}\right)$, and these groups sometimes appear as amalgamated free products $\widehat{\Gamma}_{1} \star_{\Phi} \widehat{\Gamma}_{2}$ where $\Phi \subset \operatorname{Isom}\left(\mathbb{H}^{n-1}\right)$ itself is a cofinite Coxeter group whose fundamental Coxeter polyhedron $F$ is a common facet of the fundamental Coxeter polyhedra $P_{1}$ and $P_{2}$ of $\Gamma_{1}$ and $\Gamma_{2}$ (see Sect. 2.2 and Figs. 3, 4, for example). Geometrically, a fundamental polyhedron for $\widehat{\Gamma}_{1} \star_{\Phi} \widehat{\Gamma}_{2}$ is the Coxeter polyhedron arising by glueing together $P_{1}$ and $P_{2}$ along their common facet $F$. In this context, the following general result of Karrass and Solitar [17, Theorem 10] will be useful.

Theorem 4 Let $G=A \star_{U} B$ be a free product with amalgamated subgroup $U$, and let $H$ be a finitely generated subgroup of $G$ containing a normal subgroup $N$ of $G$ such that $N \nless U$. Then, $H$ is of finite index in $G$ if and only if the intersection of $U$ with each conjugate of $H$ is of finite index in $U$. 


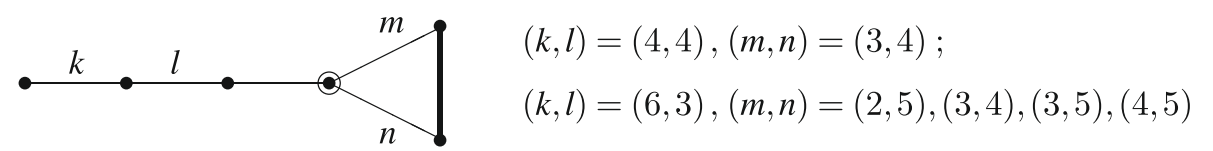

Fig. 12 The 5 non-arithmetic Coxeter pyramids $[k, l, 3,(m, \infty, n)]$ in $\mathbb{H}^{4}$

\section{The commensurability classification}

\subsection{The non-arithmetic case}

For hyperbolic Coxeter pyramid groups $\Gamma \subset \operatorname{Isom}\left(\mathbb{H}^{n}\right)$ for $n>4$, Vinberg's criterion (see Sect. 2.1) and the check for finite index subgroups (see Sect. 2.2 and [12, Chapter 5]) imply that there is only one non-arithmetic example in dimensions $n=5,6$ and 10 up to finite index, respectively, and they are all free products with an amalgamating hyperbolic Coxeter simplex subgroup in Isom $\left(\mathbb{H}^{n-1}\right)$. Their Coxeter symbols are given by $\left[4,3^{1,2},(3, \infty, 4)\right]$, $\left[3,4,3^{3},(3, \infty, 4)\right]$ and $\left[3^{2,1}, 3^{6},(3, \infty, 4)\right]$, respectively (see also Fig. 3 and the "Appendix").

In dimension 4, and among the 13 groups, there is - up to finite index-a total of 5 Coxeter groups to test for commensurability. They are depicted in Fig. 12 .

For each of these groups $\Gamma$ we compute the trace of powers $c^{r}, r \geq 1$, of a Coxeter element $c=c(\Gamma)$ (see Sect. 3). It turns out that these traces in the case of fixed $(k, l)=(6,3)$ and $(m, n)=(3,4),(3,5)$ and $(4,5)$, respectively, lie in the difference set $\mathbb{Q}(\sqrt{2})-\mathbb{Q}$, $\mathbb{Q}(\sqrt{5})-\mathbb{Q}$ and $\mathbb{Q}(\sqrt{2}, \sqrt{5})-\mathbb{Q}(\sqrt{2})-\mathbb{Q}(\sqrt{5})$, respectively. Therefore, these groups are pairwise incommensurable.

The traces of $c^{r}, r \geq 1$, of the two remaining groups, given by $(k, l)=(4,4)$, $(m, n)=(3,4)$ resp. $(k, l)=(6,3),(m, n)=(2,5)$, are in $\mathbb{Q}(\sqrt{2})-\mathbb{Q}$ resp. $\mathbb{Q}(\sqrt{5})-\mathbb{Q}$. Hence, it remains to test up to commensurability two particular pairs of groups with identical difference set of traces.

We start with the pair $(m, n)=(2,5)$ and $(m, n)=(3,5)$ having $(k, l)=(6,3)$ and giving rise to the set $\mathbb{Q}(\sqrt{5})-\mathbb{Q}$. The group $\Gamma$ with $(m, n)=(3,5)$ is a free product, amalgamated by the Coxeter subgroup $\Phi=[6,3,3]$, of the group $\widehat{\Gamma}_{1}=[6,3,3,5]$ with $(m, n)=(2,5)$ and the (arithmetic) group $\widehat{\Gamma}_{2}=[6,3,3,3]$. The Coxeter polyhedron $P \subset \mathbb{H}^{4}$ of $\Gamma$ arises by glueing the fundamental Coxeter polyhedra $P_{1}$ and $P_{2}$ of $\Gamma_{1}=[6,3,3,5, \infty]$ and $\Gamma_{2}=[6,3,3,3, \infty]$ along their common facet $F=[6,3,3]$ (see also Sect. 2.2). Observe that both polyhedra $P_{1}$ and $P_{2}$ are simply truncated Coxeter orthoschemes which have been classified by Im Hof [10] and which enjoy the following algebraic property.

Lemma 1 Let $\Theta$ be a truncated Coxeter orthoscheme group in Isom $\left(\mathbb{H}^{n}\right)$ given by the Coxeter graph in Fig. 13 where $p_{1}=\infty$ for $n=3$. Denote by $q \in \partial \mathbb{H}^{n}$ the apex at infinity of the associated Coxeter polyhedron $P \subset \mathbb{H}^{n}$. Then, the stabiliser $\Theta_{q}<\operatorname{Isom}\left(\mathbb{E}^{n-1}\right)$ of q contains a translation of translational length $\delta$ equal to $2 \cos \left(\pi / p_{n}\right)$ (with respect to the Euclidean line element $d s_{0}^{2}$ on the canonical horosphere of $\mathbb{H}^{n}$ realised in $\mathbb{E}_{+}^{n}$ (see (2.7)).

Proof First, observe that the Coxeter polyhedron $P \subset \mathbb{H}^{n}$ described by Fig. 13 is a simply truncated orthoscheme in the following sense (see also [18]). Consider the polyhedral set $P^{\prime} \subset \mathbb{H}^{n}$ bounded by the hyperplanes $H_{i}, 1 \leq i \leq n+1$, with intersection behavior as given by the corresponding subgraph in Fig. 13. The region $P^{\prime}$ is of infinite volume in $\mathbb{H}^{n}$ and can be interpreted - by passing to the projective model of $\mathbb{H}^{n}$ - as the intersection of $\mathbb{H}^{n}$ with an $n$-simplex $\hat{P}$, having vertices $v_{1}, \ldots, v_{n+1}$ opposite to $H_{1}, \ldots, H_{n+1}$ such that 


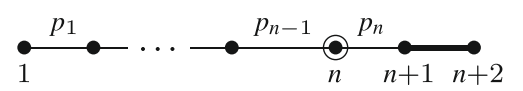

Fig. 13 The hyperbolic truncated Coxeter $n$-orthoscheme group $\left[p_{1}, \ldots, p_{n}, \infty\right]$

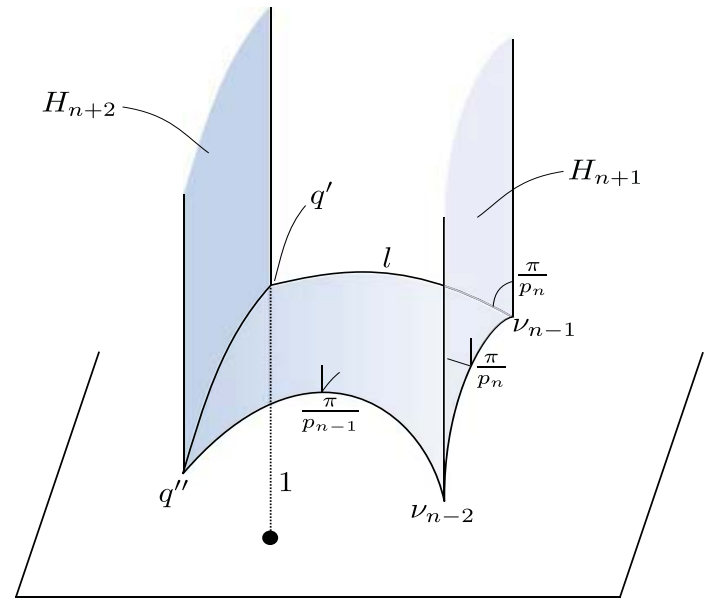

Fig. 14 The face $F$ in the upper half space model

precisely $v_{n+1}$ lies outside the defining quadric for $\mathbb{H}^{n}$ and such that the edges intersect in $\mathbb{H}^{n}$ according to $v_{i-1} v_{i} \perp v_{i} v_{i+1}$ for all $i=2, \ldots, n$ (i.e. $P^{\prime}$ is the intersection of $\mathbb{H}^{n}$ with an $n$-orthoscheme $\hat{P}$ having the ultra-ideal vertex $v_{n+1}$ ). By adjoining to $H_{1}, \ldots, H_{n+1}$ the hyperbolic (polar) hyperplane $H_{n+2}$ defined by $H_{n+2}=\left\{y \in \mathbb{H}^{n} \mid\left\langle y, v_{n+1}\right\rangle_{n, 1}=0\right\}$, the orthoscheme $\hat{P}$ will be truncated and yield the polyhedron $P$ of finite volume. The graph in Fig. 13 indicates that the hyperplanes $H_{n+1}$ and $H_{n+2}$ are parallel meeting at the apex at infinity $q:=v_{n}$ so that $P$ has the combinatorial type of a pyramid over a product of two Euclidean simplices.

Since the hyperplanes $H_{n+1}$ and $H_{n+2}$ intersect at $q \in \partial \mathbb{H}^{n}$, the composition of the associated reflections $s_{n+1}$ and $s_{n+2}$ yields the (Euclidean) translation $t=s_{n+1} \circ s_{n+2} \in \Theta_{q}$ of length $\delta$ which is equal to the double of the Euclidean distance of $H_{n+1}$ and $H_{n+2}$ (measured along a suitable horocycle). More precisely, viewing the situation in the upper half space model $\left(\mathbb{E}_{+}^{n}, d s^{2}=\left(d x_{1}^{2}+\cdots+d x_{n}^{2}\right) / x_{n}^{2}\right)$, by assuming that $q=\infty$, we can relate the length $\delta / 2$ (see (2.7)) to a hyperbolic edge length $l$ of $P$ as follows.

Consider the face $F$ formed by the vertices $v_{n-2}, v_{n-1}, v_{n}, v_{n+1}$ of $\hat{P}$ and truncated by $H_{n+2}$. Denote by $q^{\prime}$ resp. $q^{\prime \prime}$, respectively, the intersection point of the edge $v_{n-1} v_{n+1}$ resp. $v_{n-2} v_{n+1}$ with $H_{n+2}$ (see Fig. 14). Then, $F$ is a pyramid with apex $\infty$ over the Lambert quadrilateral formed by the vertices $v_{n-2}, v_{n-1}, q^{\prime}, q^{\prime \prime}$ whose non-right angle sits at $v_{n-2}$. By construction, the right-angled triangle $v_{n-1} q^{\prime} \infty$ has angle $\pi / p_{n}$ at $v_{n-1}$ so that the edge $v_{n-1} q^{\prime}$ is of hyperbolic length $l$ satisfying $\cosh l=1 / \sin \left(\pi / p_{n}\right)$.

Suppose that the face $F$ lies on the unit hemisphere centered at $0 \in \mathbb{E}^{n-1}$ such that $q^{\prime}=(0, \ldots, 0,1)$. Then, the horocyclic segment at height $x_{n}=1$ and starting from $q^{\prime}$ has (undistorted) Euclidean length $\delta / 2$ to $H_{n+1}$ which is related to $l$ by (see Fig. 15 and [3, p. 268])

$$
\delta / 2=\tanh l=\cos \frac{\pi}{p_{n}} .
$$

Remark 1 By (4.1), the translational length $\delta$ is an algebraic integer. 


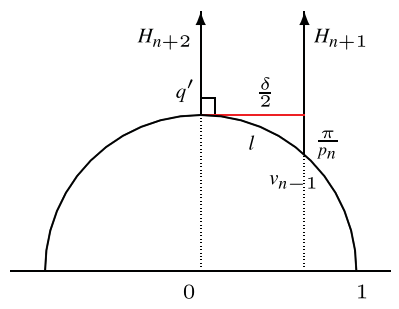

Fig. 15 Horocyclic distance $\delta / 2$ and hyperbolic edge length $l$

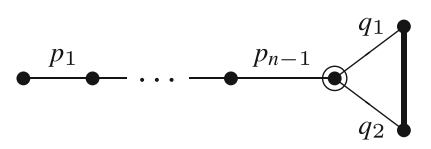

Fig. 16 The free product $\Gamma=\left[p_{1}, \ldots, p_{n-1},\left(q_{1}, \infty, q_{2}\right)\right]=\widehat{\Theta}_{1} \star_{\Phi} \widehat{\Theta}_{2}$ amalgamated by $\Phi=$ $\left[p_{1}, \ldots, p_{n-1}\right]$

By means of Lemma 1, we can derive the following simple commensurability statement for certain amalgamated free products and its factors as given by Fig. 16.

Proposition 1 Let $\Gamma$ be a hyperbolic Coxeter pyramid group with $n+2$ generators such that $\Gamma$ is the free product of the Coxeter orthoscheme groups $\widehat{\Theta}_{1}=\left[p_{1}, \ldots, p_{n-1}, q_{1}\right]$ and $\widehat{\Theta}_{2}=$ $\left[p_{1}, \ldots, p_{n-1}, q_{2}\right]$ amalgamated by their common Coxeter subgroup $\Phi=\left[p_{1}, \ldots, p_{n-1}\right]$, where $p_{1}=\infty$ for $n=3$. Suppose that $\mathbb{H}^{n} / \Gamma$ is 1-cusped. Then, the following holds.

(1) If $q_{1}=q_{2}=: q$ and $\Theta:=\left[p_{1}, \ldots, p_{n-1}, q, \infty\right]$, then $\Gamma$ is a subgroup of index 2 in $\Theta$.

(2) If $q_{1} \neq q_{2}$, then $\Gamma$ is incommensurable to $\Theta_{k}:=\left[p_{1}, \ldots, p_{n-1}, q_{k}, \infty\right]$ for $k=1$ and $k=2$.

Proof Part (1) is an immediate consequence from the geometric fact that the Coxeter pyramid $P \subset \mathbb{H}^{n}$ associated to the group $\Gamma$ arises by glueing together two isometric copies, where $q=q_{1}=q_{2}$, of the truncated Coxeter orthoscheme $P_{q}=\left[p_{1}, \ldots, p_{n-1}, q, \infty\right]$ along the orthogonal facet $F=\left[p_{1}, \ldots, p_{n-1}\right]$.

Part (2) can be dealt with as follows. Glue the two truncated Coxeter orthoschemes $P_{1}=$ $\left[p_{1}, \ldots, p_{n-1}, q_{1}, \infty\right]$ and $P_{2}=\left[p_{1}, \ldots, p_{n-1}, q_{2}, \infty\right]$ along their common facet $F=$ $\left[p_{1}, \ldots, p_{n-1}\right]$ to obtain the Coxeter pyramid $P$ with apex $\infty$ in the upper half space model $\mathbb{E}_{+}^{n}$ of $\mathbb{H}^{n}$. By assumption, $P$ does not have further vertices on the boundary $\mathbb{E}^{n-1}$. Moreover, the facet $F$ arises as intersection of the (in fact identical) polar hyperplanes $H_{n+2}^{1}$ resp. $H_{n+2}^{2}$ with $\widehat{P}_{1}$ resp. $\widehat{P}_{2}$; it shares the vertex $\infty$ with $P$ and is orthogonal to all hyperplanes apart from (but parallel to) $H_{n+1}^{1}$ resp. $H_{n+1}^{2}$ of $P_{1}$ resp. $P_{2}$ (see the proof of Lemma 1). Denote by $s_{i}^{k}$ the reflection with respect to $H_{i}^{k}$ for $i=n+1, n+2$ and $k=1,2$, and let $N_{k}=\left\langle s_{n+2}^{k}\right\rangle$ be the normaliser of $s_{n+2}^{k}$ in $\widehat{\Theta}_{k}$. Then, we have that $\Theta_{k}=\widehat{\Theta}_{k} \ltimes N_{k}$ for $k=1,2$.

Now, in each of the groups $\Theta_{1}, \Theta_{2}$ and $\Gamma$, respectively, the stabiliser of $\infty$ contains the (Euclidean and identically oriented) translation $t_{1}=s_{n+2}^{1} \circ s_{n+1}^{1}, t_{2}=s_{n+1}^{2} \circ s_{n+2}^{2}$ and $t=s_{n+1}^{2} \circ s_{n+1}^{1}$, respectively, along the same horocycle $\sigma$ at height 1 from $\mathbb{E}^{n-1}$ and of translational distance $\delta_{1}, \delta_{2}$ and $\delta$, say. Together with Lemma 1 , and since $q_{1} \neq q_{2}$, we get

$$
\delta=\delta_{1}+\delta_{2}, \quad \text { where } \delta_{i}=2 \cos \frac{\pi}{q_{i}}, \quad i=1,2, \quad \text { with } \quad \delta_{1} \neq \delta_{2}
$$

By the classification result of [10] and by the single cusp hypothesis, one gets $q_{i} \in\{3,4,5\}$ for $i=1,2$. 
Suppose that $\Gamma$ and $\Theta_{i}$ for $i=1$ or 2 are commensurable. In our situation, this means that there is a subgroup $H$ of $\Gamma$ and of $\Theta_{i}$ which is of finite index in both of them. In particular, $H$ is a non-cocompact discrete subgroup in $\operatorname{Isom}\left(\mathbb{H}^{n}\right)$ of finite covolume with non-trivial stabiliser $H_{\infty}$ and full rank translational lattice $\Lambda_{H} \subset H_{\infty}$ of finite index. Consider again the translations $t \in \Gamma$ and $t_{i} \in \Theta_{i}$ along the horocycle $\sigma$ associated to $\infty$. In fact, the subgroup $H$ must contain some integral powers $t^{k}$ and $t_{i}^{k_{i}}$, since $H$ is of finite index in $\Gamma$ and in $\Theta_{i}$. Therefore, $t^{k}$ and $t_{i}^{k_{i}}$ are both translations along $\sigma$ in the lattice $\Lambda_{H}$ which implies that $\delta$ is a rational multiple of $\delta_{i}$. This is a contradiction to (4.2) and to the fact that $q_{i} \in\{3,4,5\}$.

As a consequence of the above Proposition 1 , the two groups $\Gamma=[6,3,3,(3, \infty, 5)]$ and $\Gamma_{1}=[6,3,3,5, \infty]$ having the same difference set of traces are not commensurable.

The remaining pair consists of the group $\Gamma_{1}$ with $(k, l)=(4,4),(m, n)=(3,4)$, and which gives rise to a 2-cusped quotient space, and of the group $\Gamma_{2}$ with $(k, l)=(6,3)$, $(m, n)=(3,4)$, with a 1-cusped quotient space (see Fig. 12). Therefore, Proposition 1 does not apply. Nevertheless, the groups $\Gamma_{1}=[4,4,3,(3, \infty, 4)]$ and $\Gamma_{2}=[6,3,3,(3, \infty, 4)]$ are free products with amalgamating 1-cusped Coxeter simplex subgroups $H_{1}=[4,4,3]$ and $H_{2}=[6,3,3]$ of Isom $\left(\mathbb{H}^{3}\right)$, respectively. Inspired by the fact that the Euclidean lattices $[4,4]$ and $[6,3 \mid$ are inequivalent, we will show the following.

Lemma 2 The non-arithmetic Coxeter pyramid groups $\Gamma_{1}=[4,4,3,(3, \infty, 4)]$ with 2 cusped quotient and $\Gamma_{2}=[6,3,3,(3, \infty, 4)]$ with 1-cusped quotient are incommensurable in $\operatorname{Isom}\left(\mathbb{H}^{4}\right)$.

Proof The fundamental polyhedra $P_{1}$ and $P_{2}$ of $\Gamma_{1}$ and $\Gamma_{2}$ are Coxeter pyramids, each of which arises by glueing two truncated Coxeter orthoschemes along their common Coxeter tetrahedral facet (and which is orthogonal to all other facets it intersects). In the following we make use of the description of $P_{1}$ and $P_{2}$ according to the proofs of Lemma 1 and Proposition 1. For example, consider $P_{1}$ in the upper half space model $\mathbb{E}_{+}^{4}$, with $\infty$ as one of the two vertices on $\partial \mathbb{H}^{4}$, and which arises by glueing together the truncated Coxeter 4orthoschemes with graphs $\Theta=[4,4,3,3, \infty]$ and $\Theta^{\prime}=[4,4,3,4, \infty]$ along their common facet $F=F\left(P_{1}\right)$. Beside $q_{0}:=\infty$, let $q_{1}=(0,0,0,1), q_{2}, q_{3}$ be the vertices of $F$ such that $q_{i} q_{i+1} \perp q_{i+1} q_{i+2}$ for $i=1,2$. In fact, $F$ is a simply asymptotic orthoscheme [4, 4, 3] in $\mathbb{H}^{3}$ whose edge lengths satisfy $\tanh q_{1} q_{2}=1 / 2$ and $\tanh q_{1} q_{3}=1 / \sqrt{2}$ (see [18]). In a similar way, the glue facet $F=F\left(P_{2}\right)$ for $P_{2}$ is a simply asymptotic orthoscheme $[6,3,3]$ in $\mathbb{H}^{3}$ whose corresponding edge lengths satisfy tanh $q_{1} q_{2}=1 / 2$ and $\tanh q_{1} q_{3}=1 / \sqrt{3}$.

Now, suppose that the groups $\Gamma_{1}$ and $\Gamma_{2}$ are commensurable. Then, the intersection $K:=$ $\Gamma_{1} \cap \Gamma_{2}^{\prime}$ is of finite index in $\Gamma_{1}$ as well as in a suitable conjugate $\Gamma_{2}^{\prime}$ of $\Gamma_{2}$. The group $K$ is a non-cocompact but cofinite discrete group in $\operatorname{Isom}\left(\mathbb{H}^{4}\right)$ with fundamental polyhedron $P_{K} \subset \mathbb{E}_{+}^{4}$ having a vertex $q \in \partial \mathbb{H}^{4}$. We are mainly interested in the geometry of a fundamental parallelepiped defined by the generating translations in the stabiliser $K_{q}$. Since isometric conjugation preserves angles and (translational) length, we may suppose without loss of generality that $q=\infty$. In this way, we can compare the polyhedra $P_{1}, P_{2}$ and $P_{K}$ with respect to the boundary of the vertex neighborhood of their common vertex $\infty$ on the horosphere $S_{\infty}$ at height 1 , say. Again, $S_{\infty}$ carries a Euclidean metric in a natural way, and the stabiliser $K_{\infty}$, acting discontinuously on $S_{\infty}$, contains a finite index translational lattice $\Lambda_{K}$ of rank 3 . As above, the lattice $\Lambda_{K}$ must contain certain iterates of the three generating translations in each of the two corresponding lattices $\Lambda_{1}$ and $\Lambda_{2}^{\prime}$ (associated to $\infty$ ) of the groups $\Gamma_{1}$ and $\Gamma_{2}^{\prime}$. In the following, we determine the geometric effect of the different generating translations in $\Lambda_{1}$ 


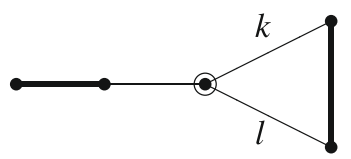

$$
\begin{aligned}
(k, l)= & (3,4),(2,5),(3,5),(4,5), \\
& (3,6),(4,6),(5,6)
\end{aligned}
$$

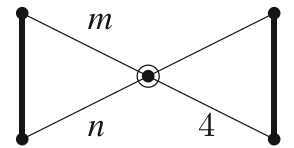

$(m, n)=(2,4),(3,4)$

Fig. 17 The 9 non-arithmetic Coxeter pyramids in $\mathbb{H}^{3}$

and $\Lambda_{2}^{\prime}$ by exploiting the particular setting of $P_{i}$ for $i=1$, 2. Firstly, observe that the graphs of $\Gamma_{1}$ and $\Gamma_{2}$ contain both the cyclic subgraph $[(3, \infty, 4)]$. The proof of Proposition 1 (see 4.2) shows that $K_{\infty}$ contains an iterate of a translation of length $\delta=2\left(\cos \frac{\pi}{3}+\cos \frac{\pi}{4}\right)=1+\sqrt{2}$, which acts along a horocycle $\sigma$ (at height 1 ) tangent to and lying above the edge through $q_{1}$ of $P_{1}$ resp. $P_{2}$. This edge is orthogonal to the glue facet [4, 4, 3] resp [6, 3, 3].

Secondly, by means of the result of Karrass and Solitar (see Theorem 4), the intersection $K_{1}:=K \cap[4,4,3]$ is of finite index in [4, 4, 3], and the intersection $K_{2}:=K \cap[6,3,3]^{\prime}$ is of finite index in $[6,3,3]^{\prime}$. Hence, the groups $K_{1}$ resp. $K_{2}$ are non-cocompact but cofinite discrete groups acting on 3-dimensional hyperbolic space with a fixed point $p_{1}$ resp. $p_{2}$ on the boundary at infinity. For $i=1,2$, denote by $L_{i} \subset K_{i}$ the translational lattice of rank 2 in the stabiliser of $p_{i}$. By construction of $K_{i}<K$, the lattice $L_{1}$ is isomorphic to the lattice induced by $[4,4]$ while the lattice $L_{2}$ is isomorphic to the lattice induced by $[6,3]$. By restricting the action of $K$ to an appropriate 2-dimensional horosphere $S_{i}$ in $\mathbb{E}_{+}^{4}$, the group $K$ must contain certain iterates of the 2 generating translations of each $L_{i}$ for $i=1,2$.

Finally, we determine the translational lengths of the 2 generators for each of the lattices $L_{1}$ and $L_{2}$ by modifying, without loss of generality, the setting in such a way that $p_{1}=p_{2}=\infty$, and that $S_{1}=S_{2}$ is the horosphere at height 1 . Consider the lattice $L_{1}$ and denote by $\delta_{1}, \delta_{2}$ the translational lengths of its (natural) generators acting along the horocycles through $q_{1}$ lying above the edges $q_{1} q_{2}$ and $q_{1} q_{3}$. Together with the horocycle $\sigma$, they form an orthant based at $q_{1}$.

By the left hand side of (4.1), $\delta_{1}=2 \tanh q_{1} q_{2}=1$ and $\delta_{2}=2 \tanh q_{1} q_{3}=\sqrt{2}$. In a similar way, the translational lengths associated to $L_{2}$ are given by 1 and $2 / \sqrt{3}$. These arithmetic facts for the four translations, that is, the translational lengths $1, \sqrt{2}$ and $2 / \sqrt{3}$ together with the translation length $1+\sqrt{2}$ of the translation $\sigma$ being pairwise incommensurable, allow us to conclude that the rank of the lattice $\Lambda_{K}$ in $K$ is 4 (and not 3). This provides the desired contradiction.

In dimension 3, there are 19 groups which yield - modulo finite index -9 groups with Coxeter graphs as given in Fig. 17 which we need to investigate with respect to commensurability.

For each of these groups $\Gamma$ we compute the trace of $c^{r}, r \geq 1$ of a Coxeter element $c=c(\Gamma)$. Since the trace of $c^{r}$ for each of the groups with $(k, l)=(3,6),(4,6),(4,5)$ and $(5,6)$ is in the difference set $\mathbb{Q}(\sqrt{3})-\mathbb{Q}, \mathbb{Q}(\sqrt{6})-\mathbb{Q}, \mathbb{Q}(\sqrt{2}, \sqrt{5})-\mathbb{Q}(\sqrt{2})-\mathbb{Q}(\sqrt{5})$ and $\mathbb{Q}(\sqrt{3}, \sqrt{5})-\mathbb{Q}(\sqrt{3})-\mathbb{Q}(\sqrt{5})$, respectively, we deduce that the groups are pairwise incommensurable.

As for the two groups $\Gamma_{k, l}$ with $(k, l)=(2,5)$ and $(3,5)$, this trace computation yields the identical difference set $\mathbb{Q}(\sqrt{5})-\mathbb{Q}$. Again, we can exploit the fact that the group $\Gamma_{3,5}$ is an amalgamated product with 1-cusped quotient space and with one factor group being 
Table 1 Commensurability invariants for $\Gamma_{m, n}^{+}$

\begin{tabular}{llll}
\hline$G$ & $\Gamma_{2,3}^{+}$ & $\Gamma_{2,4}^{+}$ & $\Gamma_{3,4}^{+}$ \\
\hline$k G$ & $\mathbb{Q}(\sqrt{2}, i)$ & $\mathbb{Q}(\sqrt{2}, i)$ & $\mathbb{Q}(\sqrt{2}, i)$ \\
$A G$ & 1 & 1 & 1
\end{tabular}

equal to the truncated Coxeter orthoscheme group $\Gamma_{2,5}$. Therefore, Proposition 1 allows us to conclude that $\Gamma_{2,5}$ and $\Gamma_{3,5}$ are not commensurable.

However, our reasoning does not help to decide about the commensurability of the remaining three groups $\Gamma_{m, n}:=\Gamma_{m, n}^{3,4}$, given by $(k, l)=(3,4)$ and $(m, n)=(2,3),(2,4)$ and $(3,4)$, and having equal trace difference set $\mathbb{Q}(\sqrt{2})-\mathbb{Q}$. By passing to the orientation preserving subgroups $G$ in $P S L(2, \mathbb{C})$, we know that the invariant trace field $k G$ and the invariant quaternion algebra $A G$ are commensurability invariants (see Sect. 3). Hence, consider the Kleinian group $\Gamma_{m, n}^{+} \subset P S L(2, \mathbb{C})$ corresponding to $\Gamma_{m, n}$. By determining the invariant trace field $k \Gamma_{m, n}^{+}$according to (3.4), we see that the commensurability class of the group $\Gamma_{3,4}^{+}$ is a singleton. More precisely, in Table 1, we summarise the results about $k G$ and $A G$ for $G=\Gamma_{m, n}^{+}$where the quaternion algebra $A G \cong M_{2}(k G)$ is taken with respect to the field $k G$.

Observe that the groups $\Gamma_{2,3}^{+}$and $\Gamma_{2,4}^{+}$have identical invariant trace field and invariant quaternion algebra. so that we cannot conclude at this stage whether they are commensurable or not. By using an argument based on the commensurator (see Sect. 3) and on some explicit volume considerations, we are able to prove that the groups $\Gamma_{2,3}$ and $\Gamma_{2,4}$ are not commensurable in the following way. Both groups are free products of two Coxeter orthoscheme groups amalgamated along a Coxeter triangle group (see Fig. 6 for the related case of $[\infty, 3,(3, \infty, 5)])$. The covolume of $\Gamma_{2, k}=[\infty, k,(3, \infty, 4)]$ equals the sum of the covolumes of the associated simply truncated Coxeter orthoscheme groups $[\infty, k, 3, \infty]$ and $[\infty, k, 4, \infty]$ for $k=3,4$. Notice that the group $[\infty, 3,4, \infty]$ is of covolume (see also (2.10))

$$
\operatorname{covol}_{3}([\infty, 3,4, \infty])=\frac{1}{8} . Л\left(\frac{\pi}{6}\right)+Л\left(\frac{5 \pi}{24}\right)-Л\left(\frac{\pi}{24}\right) .
$$

Furthermore, the group $[\infty, 3,3, \infty]$ contains the group $[\infty, 4,4, \infty]$ as a subgroup of index 3 (see 2.2). According to the volume formula for truncated orthoschemes [18, Corollary (1), p. 562], the covolume of $[\infty, 4,4, \infty]$ equals $\Omega(\pi / 4)$ so that the covolume of $[\infty, 3,3, \infty]$ is given by $\Omega(\pi / 4) / 3$. In this way, we rediscover the covolumes as given in (2.11). An accurate numerical check "indicates" that the quotient

$$
\begin{aligned}
\alpha: & =\frac{\operatorname{covol}_{3}\left(\Gamma_{2,3}\right)}{\operatorname{covol}_{3}\left(\Gamma_{2,4}\right)}=1-\frac{\frac{2}{3} Л\left(\frac{\pi}{4}\right)}{Л\left(\frac{\pi}{4}\right)+\frac{1}{8} Л\left(\frac{\pi}{6}\right)+Л\left(\frac{5 \pi}{24}\right)-Л\left(\frac{\pi}{24}\right)} \\
& \sim 0.569328
\end{aligned}
$$

is an irrational number, which suggests that the groups $\Gamma_{2,3}$ and $\Gamma_{2,4}$ are not commensurable.

Let us prove that $\alpha$ defined by (4.4) is indeed irrational implying that $\Gamma_{2,3}=$ $[\infty, 3,(3, \infty, 4)]$ and $\Gamma_{2,4}=[\infty, 4,(3, \infty, 4)]$ are incommensurable. We assume the contrary and use the fact that both groups are non-arithmetic, that is, the commensurator $C$ (see Sect. 3, (3.1)) of the groups $\Gamma_{2,3}$ and $\Gamma_{2,4}$ is a discrete subgroup of $\operatorname{Isom}\left(\mathbb{H}^{3}\right)$ containing both groups as subgroups of finite index. Observe that $C$ is a non-cocompact but cofinite (nonarithmetic) group so that its covolume is universally bounded from below by the minimal covolume $\Omega(\pi / 3) / 8$ in this class which is realised by the tetrahedral group [3, 3, 6] (see [25] and [19, Table 2]). This allows us to rewrite (4.4) according to 
Table 2 Commensurability classes $\mathcal{N}_{n}$ in the non-arithmetic case

\begin{tabular}{lllll}
\hline$n$ & $v_{n}=1$ & $v_{n}=2$ & $v_{n}=3$ & $v_{n}=4$ \\
\hline 3 & {$[(3, \infty, 4),(3, \infty, 4)]$} & {$[\infty, 3,(3, \infty, k)]$ for $k=4,5,6$} & & {$[\infty, 3,5, \infty]$} \\
& & {$[\infty, 3,(l, \infty, m)]$ for $4 \leq l<m \leq 6$} & \\
& {$[\infty, 4,(3, \infty, 4)]$} & \\
4 & & {$\left[6,3^{2},(k, \infty, l)\right]$ for $3 \leq k<l \leq 5$} & {$\left[4^{2}, 3,(3, \infty, 4)\right]$} & {$\left[6,3^{2}, 5, \infty\right]$} \\
5 & & {$\left[4,3^{2,1},(3, \infty, 4)\right]$} & & \\
6 & & & {$\left[3,4,3^{3},(3, \infty, 4)\right]$} & \\
10 & {$\left[3^{2,1}, 3^{6},(3, \infty, 4)\right]$} & & & \\
& & &
\end{tabular}

$$
\alpha=\frac{\operatorname{covol}_{3}\left(\Gamma_{2,3}\right)}{\operatorname{covol}_{3}\left(\Gamma_{2,4}\right)}=\frac{\operatorname{covol}_{3}\left(\Gamma_{2,3}\right) / \operatorname{covol}_{3}(C)}{\operatorname{covol}_{3}\left(\Gamma_{2,4}\right) / \operatorname{covol}_{3}(C)}=\frac{\left[C: \Gamma_{2,3}\right]}{\left[C: \Gamma_{2,4}\right]} .
$$

By accurate numerical computations with the softwares MATHEMATICA ${ }^{\circledR} 10$ and GP/PARI 2.7.4 we find

$$
\begin{aligned}
\operatorname{covol}_{3}\left(\Gamma_{2,4}\right)= & Л\left(\frac{\pi}{4}\right)+\frac{1}{8} Л\left(\frac{\pi}{6}\right)+Л\left(\frac{5 \pi}{24}\right)-Л\left(\frac{\pi}{24}\right) \sim 0.708943, \\
& \operatorname{covol}_{3}([3,3,6])=\frac{1}{8} . Л(\pi / 3) \sim 0.042289,
\end{aligned}
$$

so that $\left[C: \Gamma_{2,4}\right]<17$. In this way, it is easy to check that there is no rational solution $\alpha$ to (4.5) with an approximate value $\alpha \sim 0.569328$. This provides the desired contradiction.

Our results can be summarised as follows.

Theorem 1 (Commensurability classes of non-arithmetic hyperbolic Coxeter pyramid groups) Let $\Gamma \subset$ Isom $\left(\mathbb{H}^{n}\right)$ be one among the 38 non-arithmetic Coxeter pyramid groups with $n+2$ generators. Then, it belongs to one of the commensurability classes $\mathcal{N}_{n}$ given by representatives and cardinalities $v_{n}=\left|\mathcal{N}_{n}\right|$ according to Table 2.

Remark 2 The commensurability class $\mathcal{N}_{6}$ of cardinality 3 consists of the finite index subgroup sequence

$$
\left[3^{1,1,1}, 3^{2},(3, \infty, 4)\right]<2\left[4,3,3^{1,2},(3, \infty, 4)\right]<3\left[3,4,3^{3},(3, \infty, 4)\right] .
$$

\subsection{The arithmetic case}

For $n=2$, there is only one commensurability class of non-cocompact but cofinite planar hyperbolic Coxeter groups (cf. [28]). As a consequence, the two Coxeter triangle groups depicted in Fig. 18 are commensurable.

For $n=3$, similar algebraic methods as in the planar case can be developped to characterise commensurable Kleinian groups $G \subset P S L(2, \mathbb{C})$. Due to work of Maclachlan and Reid [23,

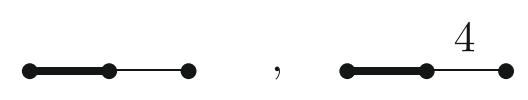

Fig. 18 Two commensurable non-compact Coxeter triangles 
Chapter 3.3], the invariant trace field $k G$ and the invariant quaternion algebra $A G$ over $k G$ (see Section 3.1) of an arithmetic cofinite Kleinian group $G \subset P S L(2, \mathbb{C})$ form a complete set of commensurability invariants for $G$ (see [23, Theorem 8.4.1]). For further computational aspects of $k G$ and $A G$, we refer to [22,23] and [1] (see also (3.4)).

For $n \geq 3$, Maclachlan [21] extended the methods and proved explicit criteria for two discrete arithmetic groups in Isom $\left(\mathbb{H}^{n}\right)$ to be commensurable (in the wide sense). As an illustration, he derived that there are exactly two commensurability classes of arithmetic Coxeter groups of rank at most 6 acting cocompactly on $\mathbb{H}^{4}$, and that the three remaining arithmetic Coxeter groups of rank 7 acting cocompactly on $\mathbb{H}^{5}$ (characterised by fundamental simplicial prisms) are pairwise incommensurable (see [21, Tables 1 and 3]). Observe thatby results of Esselmann [5] and Kaplinskaja [16] — there are no hyperbolic Coxeter groups of rank $n+2$ acting cocompactly on $\mathbb{H}^{n}$ for $n \geq 6$.

In the sequel, we shall apply the different results of Reid and Maclachlan for the arithmetic hyperbolic Coxeter pyramid groups according to [22, Theorem 3.1] and [21, Chapter 9]. We describe the procedure in detail in the case of one example (see the proof of Lemma 3 below) and summarise the complete commensurability results at the end of this section. An important ingredient is the arithmeticity criterion for non-cocompact hyperbolic Coxeter groups due to Vinberg (see Sect. 2.1).

Let $\Gamma \subset P O(n, 1)$ be an arithmetic hyperbolic Coxeter pyramid group with fundamental Coxeter pyramid $P \subset \mathbb{H}^{n}$, described by outer normal vectors $e_{1}, \ldots, e_{n+2}$, and with associated Gram matrix $G=\left(\left\langle e_{i}, e_{k}\right\rangle_{n, 1}\right)$. Then, the field of definition $k$ for $\Gamma$, generated by all the cycles of $2 G$ over $\mathbb{Q}$, is equal to $\mathbb{Q}$. Due to this fact, we shall work with cycles $g_{i_{1} i_{2}} g_{i_{2} i_{3}} \cdots g_{i_{l-1} i_{l}} g_{i_{l} i_{1}}$ of the matrix $G$ (and not $2 G$ ) (compare with (2.7) and also (3.5)) and form the new vectors

$$
v_{i_{1} i_{2} \ldots i_{k}}=g_{1 i_{1}} g_{i_{1} i_{2}} \ldots g_{i_{k-1} i_{k}} e_{i_{k}}, \quad\left\{i_{1}, i_{2}, \ldots, i_{k}\right\} \subset\{1, \ldots, n+2\} .
$$

Consider the $\mathbb{Q}$-vector space $V$ generated by the vectors given in (4.7). Then, $V$ is of dimension $n+1$ over $\mathbb{Q}$ and — equipped with the restriction $q_{V}$ of the form $\langle\cdot, \cdot\rangle_{n, 1}$ - becomes a quadratic space of signature $(n, 1,0)$. Next, extract from the vectors in $(4.7)$ a $\mathbb{Q}$-basis $v_{1}, \ldots, v_{n+1}$ and consider their Gram matrix $G_{V}:=\left(\left\langle v_{i}, v_{k}\right\rangle_{n, 1}\right)_{1 \leq j, k \leq n+1} \in G L(n+1, \mathbb{Q})$. The diagonalisation of the matrix $G_{V}$ over $\mathbb{Q}$ yields a quadratic form denoted by $\left\langle a_{1}, \ldots, a_{n+1}\right\rangle$ with $a_{1}, \ldots, a_{n+1} \in \mathbb{Q}^{*}$. Associated to the form $\left\langle a_{1}, \ldots, a_{n+1}\right\rangle$ is the Hasse invariant $s(V)$ of $V$ in the Brauer group $\operatorname{Br}(\mathbb{Q})$ represented by the tensor product of the quaternion algebras of the form $\left(a_{i}, a_{j}\right)_{\mathbb{Q}}:=\left(\frac{a_{i}, a_{j}}{\mathbb{Q}}\right)$, that is (see [20, Chapter V] and [22, Section 2.8]),

$$
s(V)=\left[\otimes_{i<j}\left(a_{i}, a_{j}\right)_{\mathbb{Q}}\right] .
$$

In the sequel, and for the sake of simplicity, we shall write a dot for the tensor product of algebras and their classes in the Brauer group; furthermore, we will drop the mention of the defining field $\mathbb{Q}$ in the Hilbert symbol. It is known that the Hasse invariant $s(V)$ does not depend on the choice of the basis $v_{1}, \ldots, v_{n+1}$ of $V$ and the resulting diagonalisation over $\mathbb{Q}$ (see [20, Chapter V, Proposition 3.18]). Furthermore, $s(V)$ is related to the Witt invariant $c(V) \in \operatorname{Br}(\mathbb{Q})$ according to

$$
\begin{array}{ll}
n \equiv 0,1(\bmod 8): & c(V)=s(V), \\
n \equiv 2,3(\bmod 8): & c(V)=s(V) \cdot(-1,-d(V)), \\
n \equiv 4,5(\bmod 8): & c(V)=s(V) \cdot(-1,-1), \\
n \equiv 6,7(\bmod 8): & c(V)=s(V) \cdot(-1, d(V)),
\end{array}
$$


where $d(V)$ equals the determinant of $q_{V}$, i.e. $d(V)=a_{1} \ldots a_{n+1}$. The invariants $s(V), c(V)$ can be represented by a quaternion algebra $B$ since we work over a number field (see [31, chapitre I, Théorème 2.9; chapitre III, Section 3]). Now, by the results of Maclachlan [21], the parametrising set for the commensurability class of $\Gamma$ in $P O(n, 1)$ can be obtained from the quaternion algebra $B$ representing the Hasse invariant $s(V)$, its ramification set $\operatorname{Ram}(B)$ and $d(V)$ (see [21, Corollary 7.3 and Corollary 7.5]).

When $n$ is even, the isomorphism class of $B$ is a complete invariant of the commensurability class of $\Gamma$ (see [21, Theorem 7.2]). Since two quaternion algebras over a number field are isomorphic if and only if their ramification sets are the same (see [20]), the problem reduces to the computation of $\operatorname{Ram}(B)=\operatorname{Ram}_{f}(B) \cup \operatorname{Ram}_{\infty}(B)$. Since $\operatorname{Ram}(B)$ is finite and of even cardinality, equation (4.9) implies that

$$
\operatorname{Ram}(B)= \begin{cases}\operatorname{Ram}_{f}(B) & \text { if } n \equiv 0,2(\bmod 8) \\ \operatorname{Ram}_{f}(B) \cup\{\infty\} & \text { if } n \equiv 4,6(\bmod 8)\end{cases}
$$

When $n$ is odd and greater than 3, the situation is more complicated since the quaternion algebra $B$ is not a complete invariant. In fact, a complete invariant consists of the quaternion algebra $B$, the discriminant (or signed determinant) $\delta$ of the quadratic form $q_{V}$ and a set of prime ideals $\left\{p_{1}, \ldots, p_{s}\right\}$ of $\mathbb{Q}$ which split in $\mathbb{Q}[\sqrt{\delta}]$ (see [21, Corollary 7.5]). For $n=3$, and by passing to the orientation preserving subgroup of $\Gamma$ in $P S L(2, \mathbb{C})$, we dispose of the much simpler characterisation of a complete set of commensurability invariants by means of the invariant trace field and the invariant quaternion algebra for $\Gamma$ (see above and [23]). For illustration, see also [1]. In the sequel, we treat the commensurability classification only for some selected cases with $n \leq 4$. For the complete classification, see [12, pp. 76-80].

Let $n=3$, and consider the 14 arithmetic examples among the Coxeter pyramid groups acting on $\mathbb{H}^{3}$ (see also Fig. 7). First, by using subgoup relations, we check easily that these groups fall into (at most) 3 commensurability classes $\mathcal{A}_{3}^{i}$; these families have cardinalities $\kappa=6,4$ and 4 , and are represented by the groups $[(3, \infty, 3),(3, \infty, 3)](\operatorname{resp} .[\infty, 3,3, \infty])$, $[(3, \infty, 3),(4, \infty, 4)](\operatorname{resp} .[\infty, 3,4, \infty])$ and $[(3, \infty, 3),(6, \infty, 6)](\operatorname{resp} .[\infty, 3,6, \infty])$ being of largest covolume (resp. of smallest covolume). All the subgroup relations can be worked out by taking the representing Coxeter graph as above and which is of highest internal symmetry, and by using the relevant procedure as mentionned at the end of Sect. 2.2. Observe that the group $[\infty, 4,4, \infty]$ is of index 3 in $[\infty, 3,3, \infty]$, and both groups are related to the symmetry group of an ideal right-angled regular octahedron in $\mathbb{H}^{3}$ with Schläfli symbol $\{3,4\}$. For each rotation subgroup $\Gamma^{+} \subset \operatorname{PSL}(2, \mathbb{C})$ of $[(3, \infty, 3),(k, \infty, k)]$ with $k=3,4,6$ we determine the invariant trace field $\mathbb{Q} \Gamma^{+}$(see (3.3) and (3.4)) and compare with arithmetic tetrahedral groups (see [14]); we summarise the results in Table 3 by providing a representative $\Gamma^{+}$and the representative of minimal covolume denoted by $\Gamma_{\text {min }}^{+}$. As a consequence, the groups $[(3, \infty, 3),(k, \infty, k)]$ with $k=3,4,6$ are pairwise incommensurable. In this way, we obtain a complete picture of the commensurability classes of arithmetic 3dimensional hyperbolic Coxeter pyramid groups and observe that there is precisely one class not representable by a tetrahedral Coxeter group; it contains the group $[\infty, 3,4, \infty]$.

Let $n=4$, and consider the 24 arithmetic Coxeter pyramid groups acting on $\mathbb{H}^{4}$. As for $n=3$, we first determine the obvious subgroup relations and see that the groups fall into 5 corresponding families of cardinality $\kappa=4$ or 6 , represented by the groups $\Gamma$ (resp. $\Gamma_{\min }$ ) with most symmetric Coxeter symbol (resp. of smallest covolume) according to Table 4 (see also Fig. 9). For each of these groups $\Gamma$ (or, more conveniently, for $\Gamma_{\min }$ ), we determine and list the complete commensurability invariant as given by the ramification set $\operatorname{Ram}(B)$ of the 
Table $3 \Gamma^{+}$and $\Gamma_{\min }^{+}$in the class $\mathcal{A}_{3}^{i}, i=1,2,3$, with invariant trace field $\mathbb{Q} \Gamma^{+}$

\begin{tabular}{llll}
\hline$\Gamma^{+}$ & $\Gamma_{\text {min }}^{+}$ & $\mathbb{Q} \Gamma^{+}$ & $\kappa$ \\
\hline$[(3, \infty, 3),(3, \infty, 3)]^{+}$ & {$[3,4,4]^{+}$} & $\mathbb{Q}(\sqrt{-1})$ & 6 \\
{$[(3, \infty, 3),(4, \infty, 4)]^{+}$} & {$[\infty, 3,4, \infty]^{+}$} & $\mathbb{Q}(\sqrt{-2})$ & 4 \\
{$[(3, \infty, 3),(6, \infty, 6)]^{+}$} & {$[3,3,6]^{+}$} & $\mathbb{Q}(\sqrt{-3})$ & 4 \\
\hline
\end{tabular}

Table 4 Two classes $\mathcal{A}_{4}^{i}$ with ramification set $\{i+1, \infty\}$ for $i=1,2$

\begin{tabular}{llll}
\hline$\Gamma$ & $\Gamma_{\text {min }}$ & $\operatorname{Ram}(B)$ & $\kappa$ \\
\hline$\left[3^{[3]}, 3,3^{[3]}\right]$ & {$[6,3,3,3, \infty]$} & $\{3, \infty\}$ & 4 \\
{$\left[3^{[3]}, 4,3^{[3]}\right]$} & {$[6,3,4,3, \infty]$} & $\{2, \infty\}$ & 4 \\
{$\left[3^{[3]}, 3,(4,3,4)\right]$} & {$[6,3,3,4, \infty]$} & $\{2, \infty\}$ & 4 \\
{$\left[\left(3,4^{2}, 3\right),(3, \infty, 3)\right]$} & {$[4,4,3,3, \infty]$} & $\{2, \infty\}$ & 6 \\
{$\left[\left(3,4^{2}, 3\right),(4, \infty, 4)\right]$} & {$[4,4,3,4, \infty]$} & $\{2, \infty\}$ & 6 \\
\hline
\end{tabular}

associated quaternion algebra $B$. As a result, we have precisely 2 pyramidal commensurability classes $\mathcal{A}_{4}^{1}$ and $\mathcal{A}_{4}^{2}$ of cardinality 4 and 20 .

Recall that the (non-cocompact) arithmetic Coxeter group $\widetilde{\Gamma}$ as given by Fig. 5, and whose Coxeter polyhedron is combinatorially a product of 2 triangles, is arithmetic as well.

Lemma 3 The Coxeter group $\widetilde{\Gamma} \subset$ Isom $\left(\mathbb{H}^{4}\right)$ depicted in Fig. 5 is commensurable to the Coxeter pyramid group $[4,4,3,3, \infty]$ and to each non-cocompact arithmetic Coxeter simplex group acting on $\mathbb{H}^{4}$.

Proof First, observe that it suffices to prove the commensurability of the groups [4, 4, 3, 3, $\infty]$ and $\widetilde{\Gamma}$ (see also Fig. 5). Indeed, the group [4, 4, 3, 3, $\infty$ ] is an index 3 subgroup of the simplex group [3, 4, 3, 4] (see Sect. 2.2), which in turn represents the single commensurability class containing all 9 non-cocompact arithmetic hyperbolic Coxeter simplex groups in $\operatorname{Isom}\left(\mathbb{H}^{4}\right)$ (see [14, Theorem 4]).

Next, by following [21, Corollary 7.3 and Section 9], we check in detail the features of the group $\widetilde{\Gamma}$ in order to conclude its commensurability with the group $[4,4,3,3, \infty]$. To this end, consider a Coxeter fundamental polyhedron $P \subset \mathbb{H}^{4}$ of $\widetilde{\Gamma}$, bounded by 6 hyperplanes $H_{i}$, with outer normal vectors $e_{i}$ of Lorentzian norm 1, and satisfying the intersection behavior as encoded by its Coxeter graph. The resulting Gram matrix $G=\left(\left\langle e_{i}, e_{k}\right\rangle_{4,1}\right)$ is of the following form.

$$
G=\left(\begin{array}{rrrrrr}
1 & -\frac{1}{\sqrt{2}} & 0 & 0 & 0 & -\frac{1}{\sqrt{2}} \\
-\frac{1}{\sqrt{2}} & 1 & -\frac{1}{2} & 0 & 0 & -\frac{1}{2} \\
0 & -\frac{1}{2} & 1 & -\frac{1}{\sqrt{2}} & -\frac{1}{2} & 0 \\
0 & 0 & -\frac{1}{\sqrt{2}} & 1 & -\frac{1}{\sqrt{2}} & 0 \\
0 & 0 & -\frac{1}{2} & -\frac{1}{\sqrt{2}} & 1 & -\frac{1}{2} \\
-\frac{1}{\sqrt{2}} & -\frac{1}{2} & 0 & 0 & -\frac{1}{2} & 1
\end{array}\right) .
$$

It is not difficult to design explicit coordinates for the normal vectors $e_{1}, \ldots, e_{6}$ which we then arrange as row vectors in the matrix $C$. 


$$
C=\left(\begin{array}{rrrrr}
1 & 0 & 0 & 0 & 0 \\
-\frac{1}{\sqrt{2}} & \frac{1}{\sqrt{2}} & 0 & 0 & 0 \\
0 & -\frac{1}{\sqrt{2}} & \frac{1}{\sqrt{2}} & \frac{1}{2} & \frac{1}{2} \\
0 & 0 & -1 & \frac{1}{\sqrt{2}} & \frac{1}{\sqrt{2}} \\
0 & 0 & 0 & -1 & 0 \\
-\frac{1}{\sqrt{2}} & -\sqrt{2} & -\frac{1}{\sqrt{2}} & \frac{1}{2} & \frac{3}{2}
\end{array}\right) .
$$

Now, we find a basis of the $\mathbb{Q}$-vector space spanned by the cyclic product vectors denoted by $v_{1}, \ldots, v_{6}($ see $(4.7))$. More concretely,

$$
v_{1}=e_{1}, \quad v_{2}=\sqrt{2} e_{2}, \quad v_{3}=\sqrt{2} e_{3}, \quad v_{4}=e_{4}, \quad v_{5}=\sqrt{2} e_{5}, \quad v_{6}=\sqrt{2} e_{6} .
$$

Hence, the vectors $v_{1}, \ldots, v_{5}$ form the desired basis and yield the new Lorentzian matrix $G_{V}=\left(\left\langle v_{i}, v_{k}\right\rangle_{4,1}\right)$ of signature $(4,1)$.

\begin{tabular}{|c|c|c|c|c|}
\hline$n$ & $\mathcal{A}_{n}^{1} \div \alpha_{n}^{1}$ & $\mathcal{A}_{n}^{2} \div \alpha_{n}^{2}$ & $\mathcal{A}_{n}^{3} \div \alpha_{n}^{3}$ & $\mathcal{A}_{n}^{4} \div \alpha_{n}^{4}$ \\
\hline \multirow[t]{2}{*}{3} & {$[(3, \infty, 3),(4, \infty, 4)]$} & {$[(3, \infty, 3),(6, \infty, 6)]$} & {$[(3, \infty, 3),(3, \infty, 3)]$} & \\
\hline & 4 & 4 & 6 & \\
\hline \multirow[t]{2}{*}{4} & {$[6,3,3,3, \infty]$} & {$[4,4,3,3, \infty]$} & & \\
\hline & 4 & 20 & & \\
\hline \multirow[t]{2}{*}{5} & {$\left[3^{[3]}, 3^{2}, 3^{[3]}\right]$} & {$\left[3^{[4]}, 3,(3, \infty, 3)\right]$} & {$\left[\left(3,4^{2}, 3\right), 3,3^{[3]}\right]$} & {$\left[\left(3,4^{2}, 3\right),\left(3,4^{2}, 3\right)\right]$} \\
\hline & 3 & 4 & 6 & 20 \\
\hline \multirow[t]{2}{*}{6} & {$\left[3^{[5]}, 3,(3, \infty, 3)\right]$} & {$\left[3^{[4]}, 3^{2}, 3^{[3]}\right]$} & {$\left[3^{[4]}, 3,\left(3,4^{2}, 3\right)\right]$} & \\
\hline & 2 & 4 & 18 & \\
\hline \multirow[t]{2}{*}{7} & {$\left[3^{[5]}, 3^{2}, 3^{[3]}\right]$} & {$\left[3^{1,1}, 3^{1,2},(3, \infty, 3)\right]$} & {$\left[3^{[6]}, 3,(3, \infty, 3)\right]$} & {$\left[3^{[4]}, 3^{2}, 3^{[4]}\right]$} \\
\hline & 2 & 4 & 8 & 12 \\
\hline \multirow[t]{2}{*}{8} & {$\left[3^{2,2}, 3^{3},(3, \infty, 3)\right]$} & & & \\
\hline & 16 & & & \\
\hline \multirow[t]{2}{*}{9} & {$\left[3^{2,2}, 3^{4}, 3^{[3]}\right]$} & & & \\
\hline & 10 & & & \\
\hline \multirow[t]{2}{*}{10} & {$\left[3^{2,1}, 3^{6},(3, \infty, 3)\right]$} & & & \\
\hline & 4 & & & \\
\hline \multirow[t]{2}{*}{11} & {$\left[3^{2,1}, 3^{7},(3, \infty, 3)\right]$} & {$\left[3^{2,1}, 3^{6},\left(3,4^{2}, 3\right)\right]$} & & \\
\hline & 2 & 3 & & \\
\hline \multirow[t]{2}{*}{12} & {$\left[3^{2,1}, 3^{6}, 3^{[4]}\right]$} & & & \\
\hline & 2 & & & \\
\hline \multirow[t]{2}{*}{13} & {$\left[3^{2,1}, 3^{8}, 3^{1,1,1}\right]$} & & & \\
\hline & 3 & & & \\
\hline \multirow[t]{2}{*}{17} & {$\left[3^{2,1}, 3^{12}, 3^{1,2}\right]$} & & & \\
\hline & 1 & & & \\
\hline
\end{tabular}

Table 5 Commensurability classes $\mathcal{A}_{n}^{k}$ with representatives and cardinalities in the arithmetic case 


$$
G(V)=\left(\begin{array}{rrrrr}
1 & -1 & 0 & 0 & 0 \\
-1 & 2 & -1 & 0 & 0 \\
0 & -1 & 2 & -1 & -1 \\
0 & 0 & -1 & 1 & -1 \\
0 & 0 & -1 & -1 & 2
\end{array}\right)
$$

The diagonalisation of the quadratic form given by $G_{V}$ over $\mathbb{Q}$ induces the quadratic form $\langle-3,1,1,1,3\rangle$, and the Eqs. (4.8) and (4.9) yield for the Hasse invariant $s(V)=1$ and for the Witt invariant $c(V)=1 \cdot(-1,-1)_{\mathbb{Q}}=B$. It is well known that the ramification set of $B=(-1,-1)_{\mathbb{Q}}$ equals $\{2, \infty\}$. Since, in even dimensions, the set $\operatorname{Ram}(B)$ is a complete invariant for the commensurability class of $\widetilde{\Gamma}$, a comparison with Table 4 allows us to conclude that $\widetilde{\Gamma}$ is commensurable to the group $[4,4,3,3, \infty]$.

Theorem 2 (Commensurability classes of arithmetic hyperbolic Coxeter pyramid groups) Let $\Gamma \subset \operatorname{Isom}\left(\mathbb{H}^{n}\right), 3 \leq n \leq 17$, be one among the 162 arithmetic Coxeter pyramid groups. Then, it belongs to one of the commensurability classes $\mathcal{A}_{n}^{k}$ given by representatives and cardinalities $\alpha_{n}^{k}=\left|\mathcal{A}_{n}^{k}\right|, k \geq 1$, according to Table 5 .

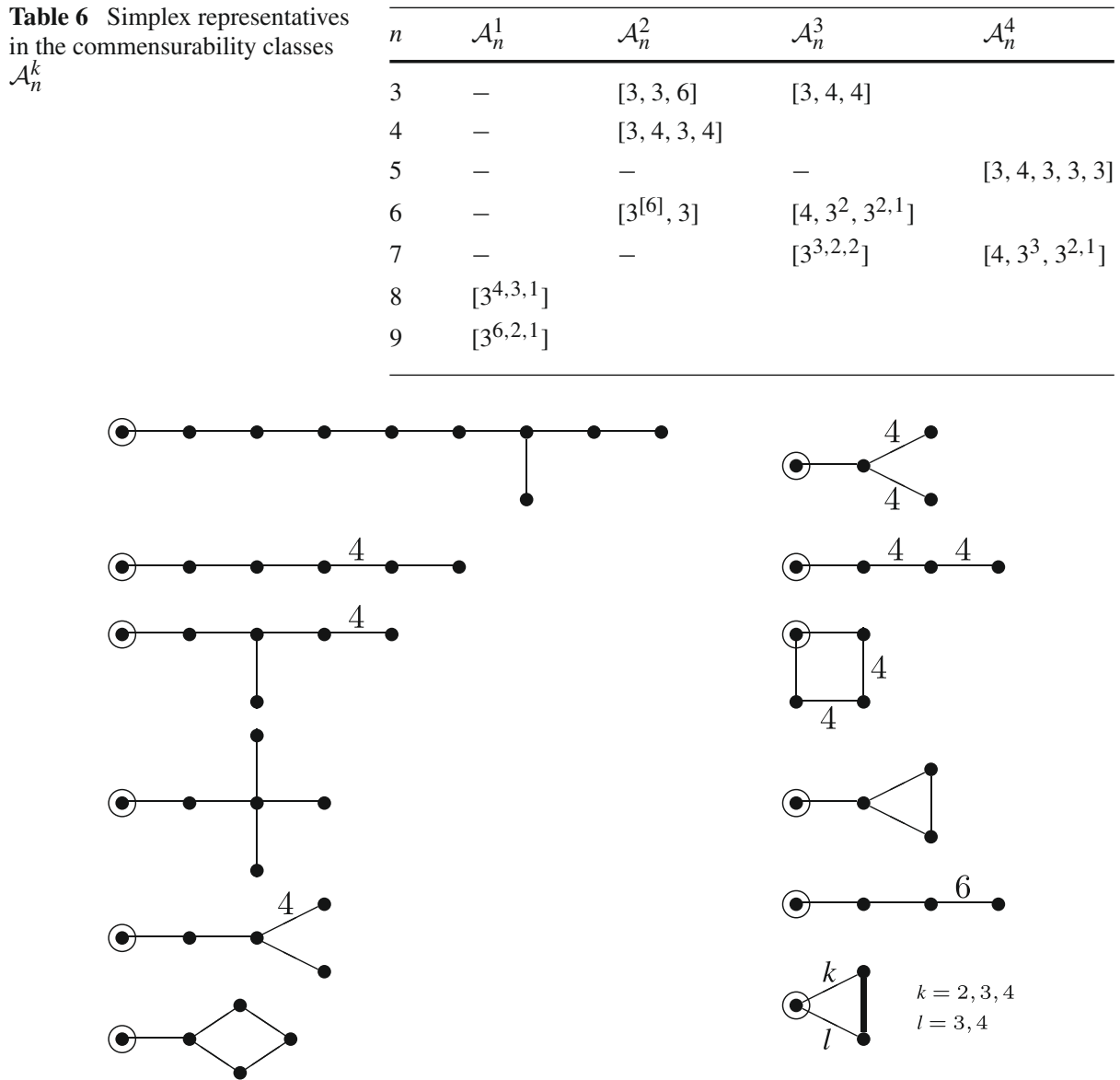

Fig. 19 Glueing together any two graphs by the encircled node yields the graph of a hyperbolic Coxeter pyramid group 

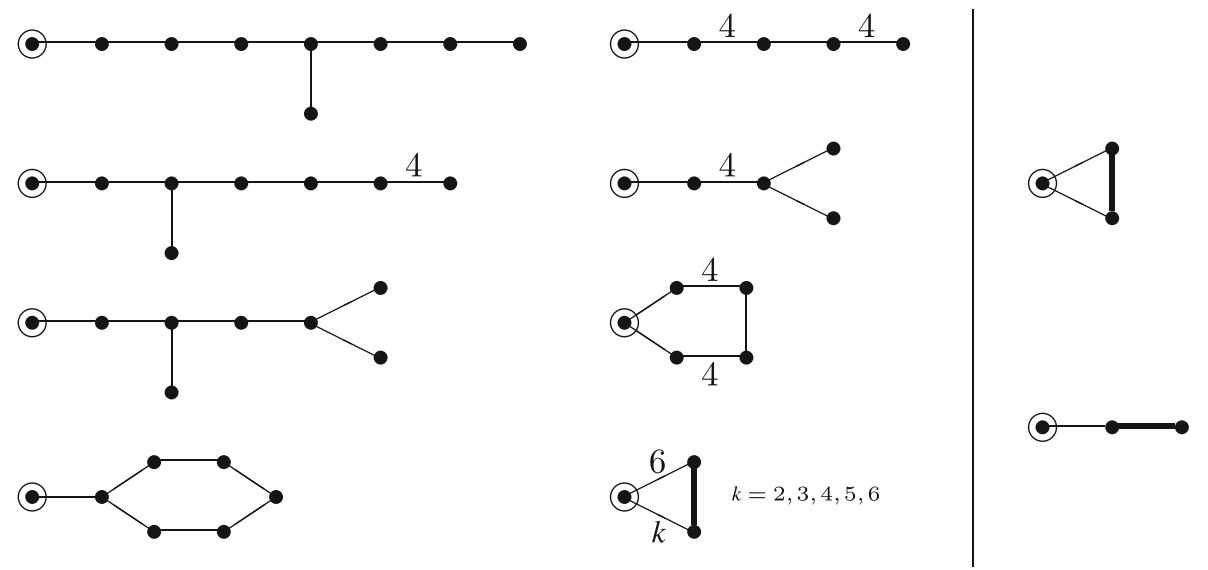

Fig. 20 Glueing together any graph from the left side with any graph from the right side by identifying the encircled node yields the graph of a hyperbolic Coxeter pyramid group
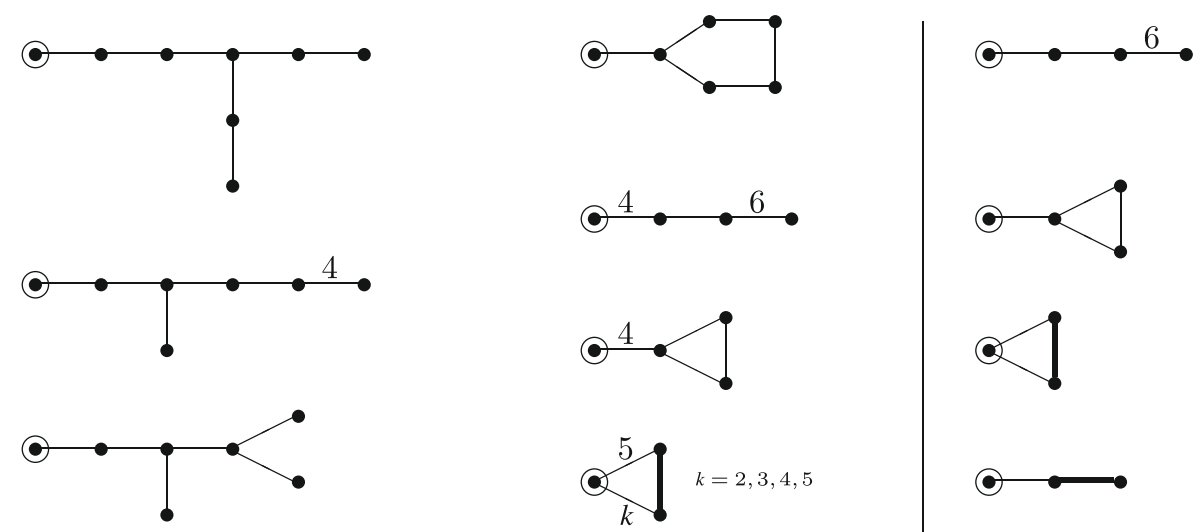

Fig. 21 Glueing together any graph from the left side with any graph from the right side by identifying the encircled node yields the graph of a hyperbolic Coxeter pyramid group

Remark 3 By applying the same method, the commensurability classification for arithmetic hyperbolic Coxeter pyramid groups can be refined by comparison with the one of the arithmetic hyperbolic simplex groups (for its list, see [14]). Notice that the latter groups exist up to dimension 9, only. Furthermore, the coincidence of the class $\mathcal{A}_{4}^{2}$ with the one of $[3,4,3,4]$ has already been proven in Lemma 3. All these results are merged in Table 6 (Figs. 19, 20, 21).

Acknowledgments The authors would like to thank Vincent Emery and John Ratcliffe for helpful discussions. The first author was fully and the second and third authors were partially supported by Schweizerischer Nationalfonds 200020-144438 and 200020-156104

\section{Appendix: Tumarkin's Coxeter pyramid groups}

The classification of the cofinite Coxeter groups in Isom $\left(\mathbb{H}^{n}\right)$ of rank $n+2$ whose fundamental polyhedra are pyramids over a product of two simplices of positive dimensions is due to Tumarkin [29,30]. The results are summarised in the Figs. 19-21 (see [30, Section 4]). 


\section{References}

1. Antolín-Camarena, O., Maloney, G., Roeder, R.: Computing arithmetic invariants for hyperbolic reflection groups. Complex Dynamics. A K Peters, Wellesley (2009)

2. Chowla, P., Chowla, S.: On irrational numbers. Nor. Vidensk. Selsk. Skr. (Trondheim) 3, 1-5 (1982)

3. Coxeter, H.S.M.: Arrangements of equal spheres in non-Euclidean spaces. Acta Math. Acad. Sci. Hung. 5, 263-274 (1954)

4. Emery, V.: Even unimodular Lorentzian lattices and hyperbolic volume. J. Reine Angew. Math. 690, 173-177 (2014)

5. Esselmann, F.: The classification of compact hyperbolic Coxeter $d$-polytopes with $d+2$ facets. Comment. Math. Helv. 71, 229-242 (1996)

6. Goodman, O., Heard, D., Hodgson, C.: Commensurators of cusped hyperbolic manifolds. Exp. Math. 17, 283-306 (2008)

7. Gromov, M., Piatetski-Shapiro, I.: Nonarithmetic groups in Lobachevsky spaces. Inst. Hautes Études Sci. Publ. Math. 66, 93-103 (1988)

8. Guglielmetti, R.: CoxIter-computing invariants of hyperbolic Coxeter groups. LMS J. Comput. Math. 18, 754-773 (2015)

9. Heckman, G.: The volume of hyperbolic Coxeter polytopes of even dimension. Indag. Math. 6, 189-196 (1995)

10. Im Hof, H.-C.: Napier cycles and hyperbolic Coxeter groups. Bull. Soc. Math. Belg. Sér. A 42, 523-545 (1990)

11. Jacquemet, M.: The inradius of a hyperbolic truncated $n$-simplex. Discrete Comput. Geom. 51, 997-1016 (2014)

12. Jacquemet, M.: New Contributions to Hyperbolic Polyhedra, Reflection Groups, and Their Commensurability, Ph.D. thesis, 2015. https://doc.rero.ch/record/257511

13. Johnson, N., Kellerhals, R., Ratcliffe, J., Tschantz, S.: The size of a hyperbolic Coxeter simplex. Transform. Groups 4, 329-353 (1999)

14. Johnson, N., Kellerhals, R., Ratcliffe, J., Tschantz, S.: Commensurability classes of hyperbolic Coxeter groups. Linear Algebra Appl. 345, 119-147 (2002)

15. Johnson, N., Weiss, A.: Quaternionic modular groups. Linear Algebra Appl. 295, 159-189 (1999)

16. Kaplinskaja, I.: The discrete groups that are generated by reflections in the faces of simplicial prisms in Lobačevskiı̌ spaces. Mat. Zametki 15, 159-164 (1974)

17. Karrass, A., Solitar, D.: The subgroups of a free product of two groups with an amalgamated subgroup. Trans. Amer. Math. Soc. 150, 227-255 (1970)

18. Kellerhals, R.: On the volume of hyperbolic polyhedra. Math. Ann. 285, 541-569 (1989)

19. Kellerhals, R.: Hyperbolic orbifolds of minimal volume. Comput. Methods Funct. Theory 14, 465-481 (2014)

20. Lam, T.Y.: Introduction to Quadratic Forms Over Fields, Graduate Studies in Mathematics, vol. 67. American Mathematical Society, Providence (2005)

21. Maclachlan, C.: Commensurability classes of discrete arithmetic hyperbolic groups. Groups Geom. Dyn. 5, 767-785 (2011)

22. Maclachlan, C., Reid, A.: Invariant trace-fields and quaternion algebras of polyhedral groups. J. Lond. Math. Soc. (2) 58, 709-722 (1998)

23. Maclachlan, C., Reid, A.: The Arithmetic of Hyperbolic 3-Manifolds, Graduate Texts in Mathematics, vol. 219. Springer, New York (2003)

24. Maxwell, G.: Euler characteristics and imbeddings of hyperbolic Coxeter groups. J. Aust. Math. Soc. Ser. A 64, 149-161 (1998)

25. Meyerhoff, R.: The cusped hyperbolic 3-orbifold of minimum volume. Bull. Am. Math. Soc. (N.S.) 13, 154-156 (1985)

26. Milnor, J.: On polylogarithms, Hurwitz zeta functions, and the Kubert identities. Enseign. Math. (2) 29, 281-322 (1983)

27. Neumann, W., Reid, A.: Arithmetic of hyperbolic manifolds. In: Topology ' 90 (Columbus, OH, 1990), Ohio State University Mathematical Research Institute Publications, vol. 1, pp. 273-310. de Gruyter. Berlin (1992)

28. Takeuchi, K.: Commensurability classes of arithmetic triangle groups. J. Fac. Sci. Univ. Tokyo Sect. IA Math. 24, 201-212 (1977)

29. Tumarkin, P.: Hyperbolic Coxeter $n$-polytopes with $n+2$ facets, p. 14. arXiv:math/0301133 (2003)

30. Tumarkin, P.: Hyperbolic Coxeter polytopes in $\mathbb{H}^{m}$ with $n+2$ hyperfacets. Mat. Zametki 75(6), 909-916 (2004) 
31. Vignéras, M.-F.: Arithmétique des algèbres de quaternions, Lecture Notes in Mathematics, vol. 800. Springer, Berlin (1980)

32. Vinberg, È.: Hyperbolic reflection groups. Russian Math. Surveys 40(1), 31-75

33. Vinberg, È: Non-arithmetic hyperbolic reflection groups in higher dimensions. In: University Bielefeld Preprint 14047 , p. 10 (2014)

34. Vinberg, E., Shvartsman, O.: Discrete groups of motions of spaces of constant curvature. In: Geometry, II, Encyclopaedia Mathematical Sciences, vol. 29, pp. 139-248. Springer, Berlin (1993) 